\title{
Executive summary of EPOS 2020 including integrated care pathways
}

Wytske J. Fokkens ${ }^{1}$, Valerie J. Lund ${ }^{2}$, Claire Hopkins ${ }^{3}$, Peter W. Hellings ${ }^{1,4}$, Robert Kern ${ }^{5}$, Sietze Reitsma', Sanna Toppila-Salmi ${ }^{6}$, Manuel BernalSprekelsen 7 , Joaquim Mullol ${ }^{8}$, on behalf of the EPOS2020 group

'Department of Otorhinolaryngology, Amsterdam University Medical Centres, location AMC, Amsterdam, The Netherlands

${ }^{2}$ Royal National Throat, Nose and Ear Hospital, UCLH, London, United Kingdom

${ }^{3}$ Ear, Nose and Throat Department, Guys and St. Thomas'Hospital, London, United Kingdom

${ }^{4}$ Department of Otorhinolaryngology, Head and Neck Surgery, University Hospitals Leuven, KU Leuven, Belgium

5 Department of Otorhinolaryngology - Head and Neck Surgery, Northwestern University, Feinberg School of Medicine, Chicago,

IL, USA

${ }^{6}$ Skin and Allergy Hospital, Helsinki University Hospital and University of Helsinki, Helsinki, Finland

${ }^{7}$ Department Hospital Quironsalud, University of Valencia, Valencia, Spain

${ }^{8}$ Rhinology Unit and Smell Clinic, ENT Department, Hospital Clínic, IDIBAPS, Universitat de Barcelona, CIBERES, Barcelona,

Catalonia, Spain
Rhinology 58: 2, 82- 111, 2020

https://doi.org/10.4193/Rhin20.601

*Received for publication:

February 1, 2020

Accepted: February 1, 2020

\subsection{Summary}

The European Position Paper on Rhinosinusitis and Nasal Polyps 2020 is the update of similar evidence based position papers published in 2005 and 2007 and $2012^{(1-3)}$. The core objective of the EPOS2020 guideline is to provide revised, up-to-date and clear evidence-based recommendations and integrated care pathways in ARS and CRS. EPOS2020 provides an update on the literature published and studies undertaken in the eight years since the EPOS2012 position paper was published and addresses areas not extensively covered in EPOS2012 such as paediatric CRS and sinus surgery. EPOS2020 also involves new stakeholders, including pharmacists and patients, and addresses new target users who have become more involved in the management and treatment of rhinosinusitis since the publication of the last EPOS document, including pharmacists, nurses, specialised care givers and indeed patients themselves, who employ increasing self-management of their condition using over the counter treatments. The document provides suggestions for future research in this area and offers updated guidance for definitions and outcome measurements in research in different settings.

EPOS2020 contains chapters on definitions and classification where we have defined a large number of terms and indicated preferred terms. A new classification of CRS into primary and secondary CRS and further division into localized and diffuse disease, based on anatomic distribution is proposed. There are extensive chapters on epidemiology and predisposing factors, inflammatory mechanisms, (differential) diagnosis of facial pain, allergic rhinitis, genetics, cystic fibrosis, aspirin exacerbated respiratory disease, immunodeficiencies, allergic fungal rhinosinusitis and the relationship between upper and lower airways. The chapters on paediatric acute and chronic rhinosinusitis are totally rewritten. All available evidence for the management of acute rhinosinusitis and chronic rhinosinusitis with or without nasal polyps in adults and children is systematically reviewed and integrated care pathways based on the evidence are proposed. Despite considerable increases in the amount of quality publications in recent years, a large number of practical clinical questions remain. It was agreed that the best way to address these was to conduct a Delphi exercise which is a structured communication technique, originally developed as a systematic, interactive forecasting method which relies on a panel of experts. The EPOS2020 group firstly prioritised the areas for consideration as a result of which we have concentrated on diagnostic issues in the first instance. The results have been integrated into the respective sections. Last but not least, advice for patients and pharmacists and a new list of research needs are included.

\subsection{Classification, definitions and terminology}

\subsubsection{Introduction}

Rhinosinusitis is a common condition in most of the world, leading to a significant burden on society in terms of healthcare consumption and productivity loss ${ }^{(4-7)}$. Acute rhinosinusitis (ARS) has a one-year prevalence of $6-15 \%$ and is usually the consequence of a viral common cold. ARS is usually a selflimiting disease but serious complications leading to life threatening situations and even death have been described ${ }^{(8)}$ It is one of the most common reasons for prescription of antibiotics and proper management is extremely pertinent in the context of the global crisis of resistance to antibiotics ${ }^{(9)}$. Chronic rhinosinusitis (CRS) is a significant health problem and affects $5-12 \%$ of the general population. The major definitions are summarized here. For more definitions please refer to chapter 2 of the EPOS2020 document. 


\subsubsection{Clinical definition of rhinosinusitis}

\subsubsection{Clinical definition of rhinosinusitis in adults}

Rhinosinusitis in adults is defined as:

- inflammation of the nose and the paranasal sinuses characterised by two or more symptoms, one of which should be either nasal blockage / obstruction / congestion or nasal discharge (anterior / posterior nasal drip):

\pm facial pain/pressure

\pm reduction or loss of smell

and either

- endoscopic signs of:

- nasal polyps, and/or

- mucopurulent discharge primarily from middle meatus and/or - oedema / mucosal obstruction primarily in middle meatus and/or

- CT changes:

- mucosal changes within the ostiomeatal complex and/or sinuses

\subsubsection{Clinical definition of rhinosinusitis in children}

Paediatric rhinosinusitis is defined as:

- $\quad$ presence of two or more symptoms one of which should be either nasal blockage / obstruction / congestion or nasal discharge (anterior / posterior nasal drip):

\pm facial pain/pressure

\pm cough

and either

- endoscopic signs of:

- nasal polyps, and/or

- mucopurulent discharge primarily from middle meatus and/or -oedema / mucosal obstruction primarily in middle meatus and/or

- $\quad$ CT changes:

-mucosal changes within the ostiomeatal complex and/or sinuses

\subsubsection{Definition for epidemiology studies and General Practice}

For epidemiological studies and general practice, the definition is based on symptomatology usually without ENT examination or radiology. We are aware that this will give an over estimation of the prevalence due to overlap with allergic and non-allergic rhinitis ${ }^{(56-58)}$

\subsubsection{Acute rhinosinusitis (ARS) in adults}

Acute rhinosinusitis in adults is defined as:

sudden onset of two or more symptoms, one of which should be either nasal blockage/obstruction/congestion or nasal discharge (anterior/posterior nasal drip):

- \pm facial pain/pressure

- $\quad \pm$ reduction or loss of smell

for $<12$ weeks;

with symptom free intervals if the problem is recurrent, with validation by telephone or interview.

\subsubsection{Acute rhinosinusitis in children}

Acute rhinosinusitis in children is defined as: sudden onset of two or more of the symptoms:

- nasal blockage/obstruction/congestion

- or discoloured nasal discharge

- or cough (daytime and night-time)

for $<12$ weeks;

with symptom free intervals if the problem is recurrent; with validation by telephone or interview.

Figure 1.2.1. Classification of primary CRS (Adapted from Grayson et al ${ }^{(154)}$ )

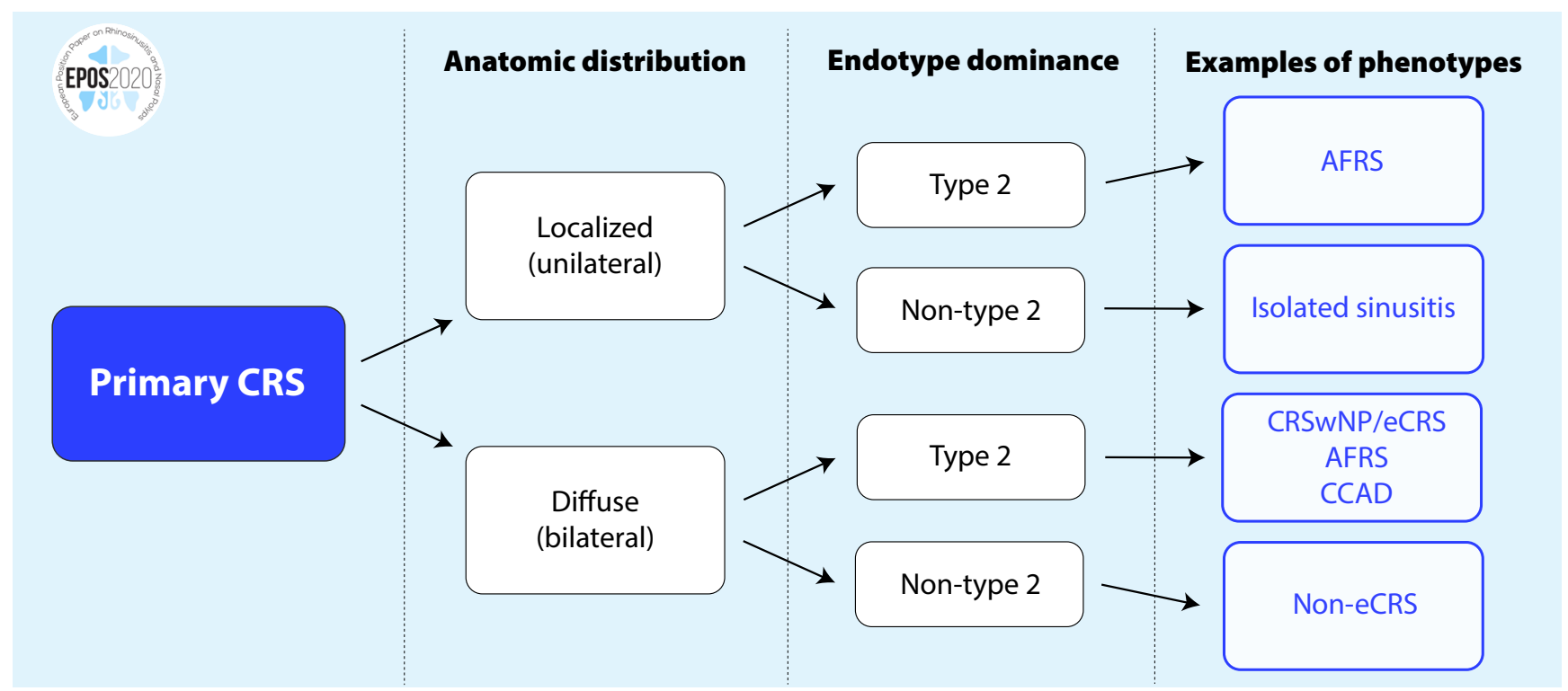

AFRS, allergic fungal rhinosinusitis; CCAD, central compartment allergic disease; CRSWNP, chronic rhinosinusitis with nasal polyps; eCRS, eosinophilic CRS. 
Questions on allergic symptoms (i.e. sneezing, watery rhinorrhoea, nasal itching, and itchy watery eyes) should be included.

\subsubsection{Recurrent acute rhinosinusitis (RARS)}

ARS can occur once or more than once in a defined time period. This is usually expressed as episodes/year but with complete resolution of symptoms between episodes.

Recurrent ARS (RARS) is defined as $\geq 4$ episodes per year with symptom free intervals ${ }^{(42,78)}$.

\subsubsection{Definition of chronic rhinosinusitis in adults}

Chronic rhinosinusitis (with or without nasal polyps) in adults is defined as:

presence of two or more symptoms, one of which should be either nasal blockage / obstruction / congestion or nasal discharge (anterior / posterior nasal drip):

- $\quad \pm$ facial pain/pressure;

- $\quad \pm$ reduction or loss of smell;

for $\geq 12$ weeks;

with validation by telephone or interview.

Questions on allergic symptoms (i.e. sneezing, watery rhinorrhoea, nasal itching, and itchy watery eyes) should be included.

\subsubsection{Definition of chronic rhinosinusitis in children}

Chronic rhinosinusitis (with or without nasal polyps) in children is defined as:

presence of two or more symptoms one of which should be either nasal blockage / obstruction / congestion or nasal discharge (anterior/posterior nasal drip):

- \pm facial pain/pressure;

- \pm cough; for $\geq 12$ weeks;

with validation by telephone or interview.

\subsubsection{Definition of difficult-to-treat rhinosinusitis}

This is defined as patients who have persistent symptoms of rhinosinusitis despite appropriate treatment (recommended medication and surgery). Although the majority of CRS patients can obtain control, some patients will not do so even with maximal medical therapy and surgery.

Patients who do not reach an acceptable level of control despite adequate surgery, intranasal corticosteroid treatment and up to two short courses of antibiotics or systemic corticosteroids in the last year can be considered to have difficult-to-treat rhinosinusitis. No changes have been made compared to EPOS2012 in the definition of severity or in acute versus chronic $^{(3)}$. For acute rhinosinusitis the term ARS comprises viral ARS (common cold) and post-viral ARS. In EPOS2007, the term 'non-viral ARS' was chosen to indicate that most cases of ARS are not bacterial. However, this term apparently led to confusion and for that reason we decided in EPOS2012 to choose the term 'post-viral ARS' to express the same phenomenon. A small percentage of the patients with post-viral ARS will have acute bacterial rhinosinusitis (ABRS).Chronic rhinosinusitis has traditionally been classified into chronic rhinosinusitis with nasal polyps (CRSwNP) and without nasal polyps (CRSsNP). CRSwNP: chronic rhinosinusitis as defined above and bilateral, endoscopically visualised polyps in middle meatus; and CRSsNP: chronic rhinosinusitis as defined above and no visible polyps in middle meatus, if necessary following decongestant.

This definition accepts that there is a spectrum of disease in CRS which includes polypoid change in the sinuses and/or

Figure 1.2.2. Classification of secondary CRS (Adapted from Grayson et al $\left.{ }^{(154)}\right)$.

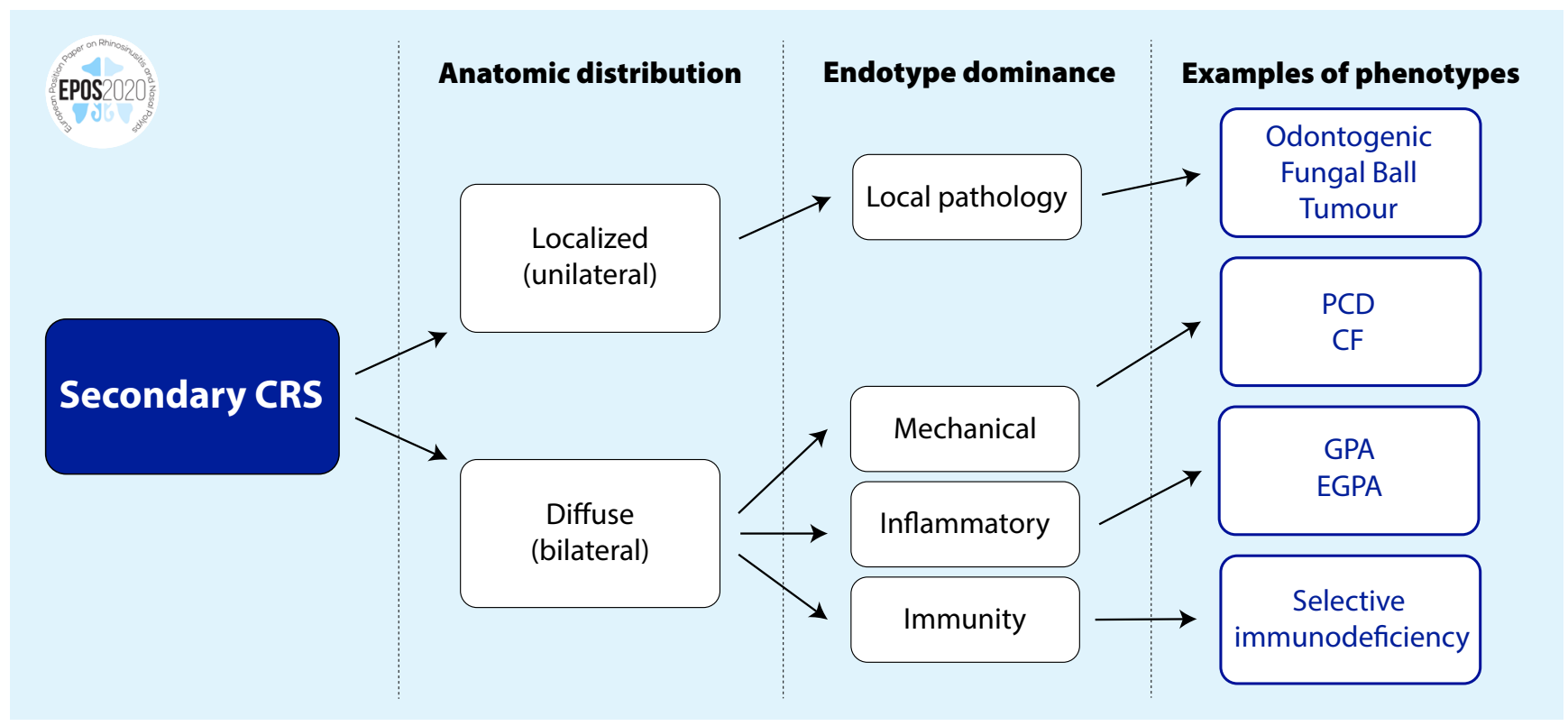

CF, cystic fibrosis; EGPA, eosinophilic granulomatosis with polyangiitis (Churg-Strauss disease); GPA, granulomatosis with polyangiitis (Wegener's disease); PCD, primary ciliary dyskinesia. 
Figure 1.2.3. Assessment of current clinical control of CRS.

\begin{tabular}{|c|c|c|c|}
\hline \multirow{2}{*}{ EPOS 2020} & \multicolumn{3}{|c|}{ EPOS 2020: Assessment of current clinical control of CRS (in the last month) } \\
\hline & $\begin{array}{l}\text { Controlled } \\
\text { (all of the following) }\end{array}$ & $\begin{array}{l}\text { Partly controlled } \\
\text { (at least } 1 \text { present) }\end{array}$ & $\begin{array}{l}\text { Uncontrolled } \\
\text { ( } 3 \text { or more present) }\end{array}$ \\
\hline Nasal blockage' & Not present or not bothersome ${ }^{2}$ & $\begin{array}{l}\text { Present } \\
\text { on most days of the week }\end{array}$ & $\begin{array}{l}\text { Present } \\
\text { on most days of the week }\end{array}$ \\
\hline Rhinorrhoea / Postnasal drip' & Little and mucous ${ }^{2}$ & $\begin{array}{l}\text { Mucopurulent } \\
\text { on most days of the week }\end{array}$ & $\begin{array}{l}\text { Mucopurulent } \\
\text { on most days of the week }\end{array}$ \\
\hline Facial pain / Pressure' & $\begin{array}{l}\text { Not present } \\
\text { or not bothersome } 2\end{array}$ & $\begin{array}{l}\text { Present } \\
\text { on most days of the week }\end{array}$ & $\begin{array}{l}\text { Present } \\
\text { on most days of the week }\end{array}$ \\
\hline Smell 1 & $\begin{array}{l}\text { Normal } \\
\text { or only slightly impaired }{ }^{2}\end{array}$ & Impaired ${ }^{3}$ & Impaired ${ }^{3}$ \\
\hline Sleep disturbance or fatigue ${ }^{1}$ & Not present ${ }^{2}$ & Present ${ }^{3}$ & Present ${ }^{3}$ \\
\hline $\begin{array}{l}\text { Nasal endoscopy } \\
\text { (if available) }\end{array}$ & $\begin{array}{l}\text { Healthy } \\
\text { or almost healthy mucosa }\end{array}$ & Diseased mucosa ${ }^{4}$ & Diseased mucosa ${ }^{4}$ \\
\hline $\begin{array}{l}\text { Rescue treatment } \\
\text { (in last } 6 \text { months) }\end{array}$ & Not needed & $\begin{array}{l}\text { Need of } 1 \text { course of } \\
\text { rescue treatment }\end{array}$ & $\begin{array}{l}\text { Symptoms (as above) persist } \\
\text { despite rescue treatment(s) }\end{array}$ \\
\hline
\end{tabular}

CRS, chronic rhinosinusitis; VAS, visual analogue scale.

middle meatus but excludes those with polypoid disease presenting in the nasal cavity to avoid overlap. Moreover, it has become progressively clear that CRS is a complex disease consisting of several disease variants with different underlying pathophysiologies ${ }^{(10,11)}$. The phenotypes do not provide full insight into all underlying cellular and molecular pathophysiologic mechanisms of CRS which becomes increasingly relevant because of the variable association with comorbidities such as asthma and responsiveness to different treatments including corticosteroids, surgery and biological agents $^{(12-15)}$. Better identification of endotypes might permit individualization of therapy that can be targeted against the pathophysiologic processes of a patient's endotype, with potential for more effective treatment and better patient outcomes.

\subsubsection{Classification of CRS}

The EPOS2020 steering group has chosen to look at CRS in terms of primary and secondary (Figures 1.2.1. and 1.2.2.) and to divide each into localized and diffuse disease based on anatomic distribution. In primary CRS, the disease is considered by endotype dominance, either type 2 or non-type 2 (see 1.5.2.2.).

Clinically localized primary CRS is then subdivided into two phenotypes - allergic fungal rhinosinusitis (AFRS) or an isolated sinusitis. For diffuse CRS, the clinical phenotypes are predominantly eCRS and non-eCRS, determined by the histologic quantification of the numbers of eosinophilic, i.e. number/high powered field which the EPOS panel agreed to be 10/hpf (400x) or higher.

For secondary CRS, again, the division is into localized or diffuse and then considered by four categories dependant on local pathology, mechanical, inflammatory and immunological factors. Thence a range of clinical phenotypes are included as shown.

There has been some discussion about a possible umbrella term of 'eosinophilic fungal rhinosinusitis' but it was agreed that 'allergic' fungal rhinosinusitis should be retained as the principle term due to common usage, recognising that not all cases have evidence of an allergic reaction to fungi e.g. a positive skin prick and/or specific lgE.

\subsubsection{Other consensus terms related to treatment}

From the many terms used regarding the sufficiency of medical treatment prior to surgery, 'appropriate medical therapy' is the preferred option of EPOS2020. Other decisions were the preferential use of the terms 'irrigation' or 'rinsing' when using saline therapy and with respect to duration of antibiotic courses, the EPOS panel also agreed that four weeks or less would be 'short-term', accepting that in general practice the duration is usually $<10$ days, and $>4$ weeks would be regarded as 'long-term'. It was also acknowledged that the aim of short-term treatment was different from long-term in that short-term courses are generally given for significant acute bacterial infection whereas long term courses are given for their immunomodulatory properties. Immunomodulation 
encompasses all therapeutic interventions aimed at modifying the immune response and is the preferred over-riding term by EPOS2020. In the treatment of rhinosinusitis, it encompasses the use of biological agents and macrolides as above.

With respect to surgery, functional implies restitution of physiology and is usually, though not exclusively, applied to endoscopic sinus surgery. It should fulfil the following criteria:

- Creates a sinus cavity that incorporates the natural ostium;

- Allows adequate sinus ventilation;

- Facilitates mucociliary clearance;

- Facilitates instillation of topical therapies.

In contrast, a 'Full FESS' is defined as complete sinus opening including anterior and posterior ethmoidectomy, middle meatal antrostomies (likely large), sphenoidotomy and frontal opening (e.g. Draf Ila ).Extended endoscopic surgery is used in the same context as 'full' (e.g. Draf III) but could also include extension beyond the confines of sinuses i.e. skull base, orbit, pterygopalatine and infratemporal fossa. Finally, radical also includes significant removal of inflamed / dysfunctional mucosa.

\subsubsection{Control of disease}

In EPOS2012 we introduced the concept of control ${ }^{(3)}$. The primary goal of any treatment, especially in chronic diseases, is to achieve and maintain clinical control, which can be defined as a disease state in which the patient does not have symptoms, or the symptoms are not impacting quality of life. In the last decade some studies have been performed that attempted to validate the EPOS2012 proposed measurement of control(15-17). Based on these validation studies, the EPOS2020 steering group thinks that the current EPOS2012 control criteria might overestimate the percentage of patients being uncontrolled. For research purposes we, therefore, recommend using a VAS scale for all symptoms: "not bothersome" can be substituted by 'VAS $\leq$ 5', and 'present / impaired' by 'VAS > 5'. Furthermore, we want to make sure that the symptoms are related to CRS and included that in the table. For example, a typical migraine headache should not be taken into account when evaluating control in CRS. The results of the validation studies also still require further psychometric validation (including internal consistency, responsiveness and known group differences) (Figure 1.2.3.). Given the importance of the concept of disease control, from a clinical as well as from a research perspective, there still remains a need for a gold standard to assess disease control in CRS.

\subsubsection{Acute exacerbation of chronic rhinosinusitis (AECRS)}

Acute exacerbation of chronic rhinosinusitis (AECRS) is defined as worsening of symptom intensity with return to baseline CRS symptom intensity, often after intervention with corticosteroids and/or antibiotics. The prevalence varies with the patient cohort being studied, season, and how the exacerbation was defined. The precise aetiology of acute exacerbation of CRS is still unclear and is likely to be multifactorial. The role of bacterial infection may have been over-emphasised in the past. Certainly, there is a lack of bacterial airway pathogens identified in the majority of patients with exacerbation. It is possible that since many of these patients have had sinus surgery in the past, postoperative changes in the microbiome create a new microbial environment and other pathogens are in play. Microbial dysbiosis in the form of an altered balance of the bacterial flora rather than a single pathogen may elicit a host inflammatory response.

Virus infections are perhaps more likely to be a key cause of exacerbation of CRS, especially with increasing evidence that

Figure 1.3.1. Prevalence of cardinal symptoms of CRS 25,26$)$.

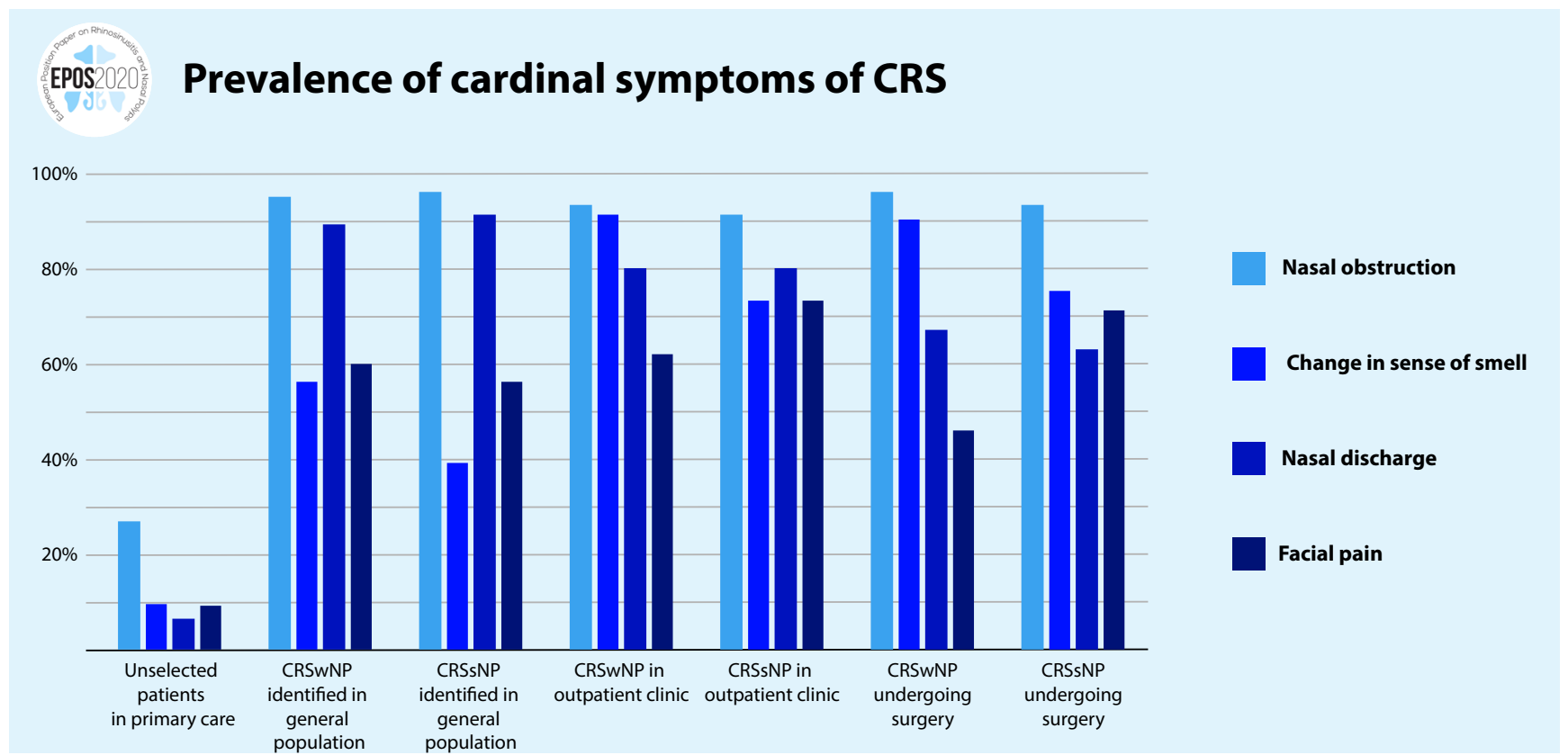

CRS, chronic rhinosinusitis; CRSsNP, chronic rhinosinusitis without nasal polyps; CRSwNP, chronic rhinosinusitis with nasal polyps. 


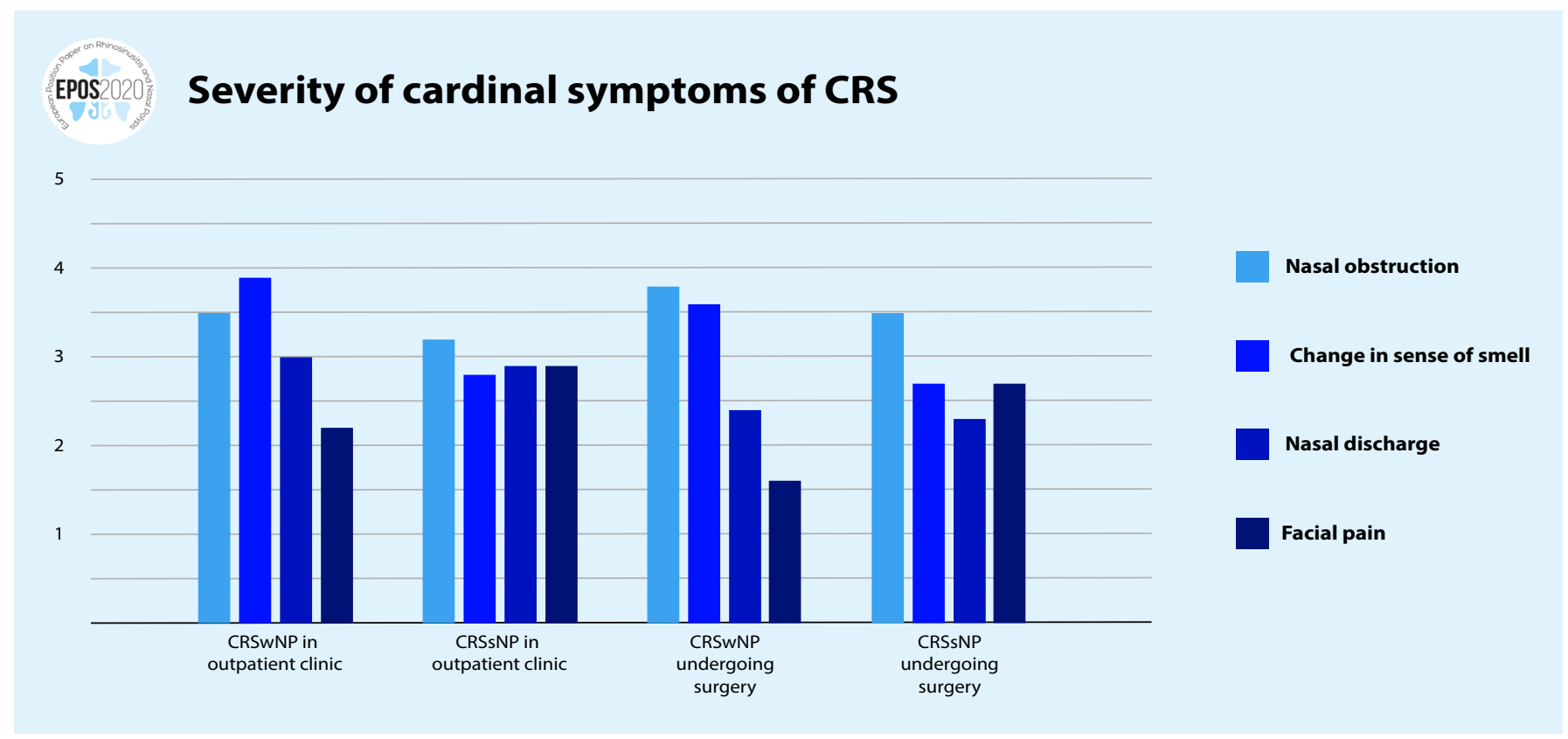

CRS, chronic rhinosinusitis; CRSsNP, chronic rhinosinusitis without nasal polyps; CRSwNP, chronic rhinosinusitis with nasal polyps.

rhinovirus infection can drive eosinophilic inflammation and a focus on prevention and management of virus infections may be more effective than treating secondary infections with antibiotics and eosinophilic flare ups with corticosteroids. However, this remains to be further investigated.

Firm scientific evidence is still lacking on therapy of AECRS and only treatment recommendations based on clinical experience and expert opinion are available. However, due to the cyclic and self-limiting nature of AECRS one should be mindful of the 'regression to the mean phenomena'. A patient is more likely to seek treatment when they are at their worst, the likelihood of improvement is high regardless of treatment, which may distort the doctor's clinical experience as well as rendering clinical trials lacking a placebo arm rather meaningless. In spite of this confounder, it is likely that steroids and antibiotics will remain the mainstay of treatment for the foreseeable future even though the role of antibiotics in the treatment of AECRS is not supported by the literature (see chapter 1.6 and 6.1).

\subsection{Burden of acute and chronic rhinosinusitis}

Chapter 3 covers the burden of rhinosinusitis, its impact on quality of life and the costs, both direct and indirect.

\subsubsection{Quality of life (QOL)}

Both ARS and CRS are associated with significant adverse effects on quality of life using a variety of validated questionnaires including the general health Eq-5D ${ }^{(18,19)}$ and SF36 $6^{(20,21)}$ and more rhinologic-specific SNOT16 $6^{(22)}$ and SNOT $22^{(23)}$. Chronic rhinosinusitis produces greater quality of life impairment than acute $^{(24)}$. Gliklich and Metson first demonstrated the impact of CRS on global quality of life, finding that CRS had a greater impact on social functioning than angina or chronic heart failure $^{(20)}$. More recently, they have shown that health utility values, measured using the EQ-5D, were lower than the general population, and comparable to other chronic diseases such as asthma ${ }^{(19)}$.

In CRS, the 'cardinal' symptoms are nasal obstruction or congestion, nasal discharge (which can be anterior or posterior), alteration in sense of smell and facial pain and pressure. These may vary in prevalence between unselected patients in primary care, CRS patients in the general population, in an outpatient setting and those undergoing surgery and in severity between those seen in outpatients and those undergoing surgery (Figure 1.3.1).

Nasal obstruction and alteration in sense of smell and taste are both the most severe and prevalent symptoms in CRSwNP, while in CRSsNP, nasal obstruction is again the most severe, with facial pain and nasal discharge reported as equally severe as altered smell and taste ${ }^{(25,26)}$ (Figure 1.3.2.). In patients presenting to ENT clinics, the presence of cardinal symptoms has a positive predictive value of 39.9 , with high sensitivity but low specificity for a diagnosis of $\mathrm{CRS}^{(27)}$.

The overall severity rating of symptoms is obviously highly dependent upon the population being studied. Patients in secondary care awaiting surgery report mean symptom severity scores in the moderate to severe range, with a mean SNOT-22 score of 42.0 compared with a control group where a mean score of 9.3 was reported ${ }^{(23)}$. CRSsNP patients had higher preoperative baseline scores (44.2) compared with CRSwNP (41.0).

\subsubsection{Costs of rhinosinusitis}

Health care spending is significantly greater in rhinosinusitis than in other diseases such as peptic ulcer disease, acute 
Table 1.4.1. Treatment evidence and recommendations for adults and children with acute viral rhinosinusitis (common cold)*.

\begin{tabular}{|c|c|c|}
\hline Therapy & $\begin{array}{l}\text { Level of } \\
\text { evidence }\end{array}$ & GRADE recommendation \\
\hline Antibiotics & $1 \mathrm{a}(-)$ & $\begin{array}{l}\text { There is no evidence of benefit from antibiotics for the common cold or for persisting acute purulent } \\
\text { rhinitis in children or adults. There is evidence that antibiotics cause significant adverse effects in adults } \\
\text { when given for the common cold and in all ages when given for acute purulent rhinitis. Routine use of } \\
\text { antibiotics for these conditions is not recommended. }\end{array}$ \\
\hline Nasal corticosteroid & $1 \mathrm{a}(-)$ & $\begin{array}{l}\text { The current evidence does not support the use of nasal corticosteroids for symptomatic relief from the } \\
\text { common cold }\end{array}$ \\
\hline Antihistamines & $1 a$ & $\begin{array}{l}\text { Antihistamines have a limited short-term (days } 1 \text { and } 2 \text { of treatment) beneficial effect on severity of over- } \\
\text { all symptoms in adults but not in the mid to long term. There is no clinically significant effect on nasal } \\
\text { obstruction, rhinorrhoea or sneezing }\end{array}$ \\
\hline Decongestant (oral / nasal) & la & $\begin{array}{l}\text { The current evidence suggests that multiple doses of decongestants may have a small positive effect on } \\
\text { subjective measures of nasal congestion in adults with the common cold. Decongestants do not seem to } \\
\text { increase the risk of adverse events in adults in the short term. }\end{array}$ \\
\hline $\begin{array}{l}\text { Paracetamol } \\
\text { (Acetaminophen) }\end{array}$ & la & $\begin{array}{l}\text { Paracetamol may help relieve nasal obstruction and rhinorrhoea but does not appear to improve other cold } \\
\text { symptoms (including sore throat, malaise, sneezing and cough) }\end{array}$ \\
\hline NSAIDs & la & $\begin{array}{l}\text { NSAIDs do not significantly reduce the total symptom score, or duration of colds. However, for outcomes } \\
\text { related to the analgesic effects of NSAIDs (headache, ear pain and muscle and joint pain) NSAIDs produce } \\
\text { significant benefits, and malaise shows a borderline benefit, although throat irritation is not improved. Chills } \\
\text { show mixed results. For respiratory symptoms, cough and nasal discharge scores are not improved, but the } \\
\text { sneezing score is significantly improved. There is no evidence of increased frequency of adverse effects in } \\
\text { the NSAID treatment groups. }\end{array}$ \\
\hline $\begin{array}{l}\text { Antihistamine-decongestant- } \\
\text { analgesic combinations }\end{array}$ & la & $\begin{array}{l}\text { Antihistamine-analgesic-decongestant combinations have some general benefit in adults and older } \\
\text { children with common cold. These benefits must be weighed against the risk of adverse effects. There is no } \\
\text { evidence of effectiveness in young children. }\end{array}$ \\
\hline Ipratropium bromide & la & $\begin{array}{l}\text { The existing evidence suggests that ipratropium bromide is likely to be effective in ameliorating } \\
\text { rhinorrhoea. Ipratropium bromide has no effect on nasal congestion and its use is associated with more } \\
\text { side effects compared to placebo or no treatment although these appeared to be well tolerated and self- } \\
\text { limiting. }\end{array}$ \\
\hline Nasal irrigation with saline & $\mathrm{lb}$ & $\begin{array}{l}\text { Nasal saline irrigation possibly has benefits for relieving the symptoms of acute URTIs mainly in children and } \\
\text { is considered an option by the EPOS steering group. }\end{array}$ \\
\hline Steam / heated humidified air & $1 \mathrm{a}(-)$ & $\begin{array}{l}\text { The current evidence does not show any benefits or harms from the use of heated, humidified air delivered } \\
\text { for the treatment of the common cold. }\end{array}$ \\
\hline Probiotics & la & $\begin{array}{l}\text { Probiotics may be more beneficial than placebo for preventing acute URTIs. However, the quality of the } \\
\text { evidence was (very) low. }\end{array}$ \\
\hline Vitamin C & la & $\begin{array}{l}\text { Given the consistent effect of vitamin C on the duration and severity of colds in regular supplementation } \\
\text { studies, and the low cost and safety, it may be worthwhile for common cold patients to test on an } \\
\text { individual basis whether therapeutic vitamin C is beneficial for them. }\end{array}$ \\
\hline Vaccines & $1 \mathrm{~b}(-)$ & $\begin{array}{l}\text { There are no conclusive results to support the use of vaccines for preventing the common cold in healthy } \\
\text { people. This is in contrast to influenza vaccines. }\end{array}$ \\
\hline Exercise & la & Regular, moderate-intensity exercise may have an effect on the prevention of the common cold. \\
\hline Echinacea & $1 \mathrm{a}(-)$ & $\begin{array}{l}\text { Echinacea products have not been shown to provide benefits for treating colds, although, there could be } \\
\text { a weak benefit from some Echinacea products: the results of individual prophylaxis trials consistently show } \\
\text { positive (if non-significant) trends, although potential effects are of questionable clinical relevance. }\end{array}$ \\
\hline Zinc & la & $\begin{array}{l}\text { Zinc administered as zinc acetate or zinc gluconate lozenges at a dose of }>=75 \mathrm{mg} / \mathrm{day} \text { and taken } \\
\text { within } 24 \text { hours of onset of symptoms significantly reduces the duration of common cold. For those } \\
\text { considering using zinc it is advised to use it at this dose throughout the cold. Regarding prophylactic zinc } \\
\text { supplementation, currently no firm recommendation can be made because of insufficient data. }\end{array}$ \\
\hline $\begin{array}{l}\text { Herbal medicine (excluding } \\
\text { Echinacae) }\end{array}$ & $\mathrm{lb}$ & $\begin{array}{l}\text { Some herbal medicines like BNO1016, Cineole and Andrographis paniculata SHA-10 extract have significant } \\
\text { impact on symptoms of common cold without important adverse events. A formal systematic review is } \\
\text { missing. }\end{array}$ \\
\hline Fusafungine & la & $\begin{array}{l}\text { Fusafungine is an effective treatment of common cold especially when administered early. However, } \\
\text { serious allergic reactions involving bronchospasm although rare have occurred after the use of fusafungine. } \\
\text { For that reason, the medication is no longer on the market. }\end{array}$ \\
\hline
\end{tabular}


Table 1.4.2. Treatment evidence and recommendations for adults with acute post-viral rhinosinusitis.

\begin{tabular}{|c|c|c|}
\hline Therapy & $\begin{array}{l}\text { Level of } \\
\text { evidence }\end{array}$ & GRADE recommendation \\
\hline Antibiotics & $1 \mathrm{a}(-)$ & $\begin{array}{l}\text { There is no benefit from prescribing antibiotics for post viral ARS in adults. There is no effect on } \\
\text { cure or duration of disease and there are more adverse events. Based on the moderate level of } \\
\text { evidence and the fact that acute post-viral rhinosinusitis is a self-limiting disease, the EPOS2020 } \\
\text { steering group advises against the use of antibiotics for adults in this situation. }\end{array}$ \\
\hline Nasal corticosteroids & $1 \mathrm{a}$ & $\begin{array}{l}\text { Nasal corticosteroids are effective in reducing total symptom score in adults suffering from } \\
\text { acute post-viral rhinosinusitis. However, the effect is small. Nasal corticosteroids have not been } \\
\text { shown to have an effect on QOL. Acute post-viral rhinosinusitis is a self-limiting disease. Based } \\
\text { on the moderate quality of the evidence and the small effect size the EPOS } 2020 \text { steering group } \\
\text { advises only to prescribe a nasal corticosteroid when reduction of the symptoms of the acute } \\
\text { post-viral rhinosinusitis is considered necessary. }\end{array}$ \\
\hline Systemic corticosteroids & $1 a$ & $\begin{array}{l}\text { Systemic corticosteroids, with or without antibiotics do not have a positive effect on recovery } \\
\text { at 7-14 days. There is a small but significant effect of systemic corticosteroids versus placebo } \\
\text { on facial pain at days } 4-7 \text { after start of the treatment. There are no studies comparing systemic } \\
\text { corticosteroids to nasal corticosteroids. The quality of the evidence is low. Based on the } \\
\text { evidence, the numbers needed to treat and the potential harm of systemic corticosteroids, } \\
\text { the EPOS2020 steering group advises against the use of systemic corticosteroids in patients } \\
\text { suffering from acute post-viral rhinosinusitis. }\end{array}$ \\
\hline Decongestant (oral / nasal) & $\mathrm{lb}$ & $\begin{array}{l}\text { Nasal decongestants may be effective in improving mucociliary clearance throughout the } \\
\text { acute phase of the disease. No studies have been performed evaluating the effect on resolution } \\
\text { or reduction of symptoms of postviral ARS. Based on the absence of clinically relevant data, } \\
\text { the EPOS2020 steering group cannot advise on the use of decongestants in acute post-viral } \\
\text { rhinosinusitis. }\end{array}$ \\
\hline Nasal irrigation with saline & $\mathrm{lb}$ & $\begin{array}{l}\text { One small study did not find a difference between saline nasal spray versus no treatment. } \\
\text { One very small study found a larger effect of high volume versus low volume saline rinsing } \\
\text { on purulent rhinorrhoea and post-nasal drip. Based on the very low quality of the evidence } \\
\text { no strong advice can be given about the use of nasal saline irrigation although on theoretical } \\
\text { grounds saline can be expected to be beneficial rather than harmful. }\end{array}$ \\
\hline Homeopathy & $\mathrm{lb}$ & $\begin{array}{l}\text { We found one study evaluating the effect of homeopathy (sinfrontal) showing a significant } \\
\text { reduction of symptoms and radiographic improvement versus placebo. Based on the limited } \\
\text { evidence the EPOS2020 steering group cannot give clear advice on the use of homeopathy in } \\
\text { acute post-viral rhinosinusitis. }\end{array}$ \\
\hline Herbal medicine & $\mathrm{lb}$ & $\begin{array}{l}\text { Some herbal medicines like BNO1016 tablets and Pelargonium sidoides drops and Myrtol } \\
\text { (and other essential oil) capsules have significant impact on symptoms of acute postviral } \\
\text { rhinosinusitis without significant adverse events. }\end{array}$ \\
\hline
\end{tabular}

ARS, acute rhinosinusitis; QOL, quality of life.

Table 1.4.3. Treatment evidence and recommendations for children with acute post-viral rhinosinusitis.

\begin{tabular}{|c|c|c|}
\hline Therapy & $\begin{array}{l}\text { Level of } \\
\text { evidence }\end{array}$ & GRADE recommendation \\
\hline Antibiotics & $1 \mathrm{a}(-)$ & $\begin{array}{l}\text { The use of antibiotics in children with acute post-viral rhinosinusitis is not associated with greater } \\
\text { cure/significant improvement. Based on the moderate level of evidence and the fact that acute } \\
\text { post-viral rhinosinusitis is a self-limiting disease, the EPOS2020 steering group advises against the } \\
\text { use of antibiotics for children in this situation. }\end{array}$ \\
\hline Nasal corticosteroids & $1 \mathrm{a}$ & $\begin{array}{l}\text { Nasal corticosteroids seem to be effective in reducing total symptom score in children } \\
\text { suffering from acute post-viral rhinosinusitis on top of (ineffective) antibiotics. Acute post- } \\
\text { viral rhinosinusitis is a self-limiting disease. Based on the very low quality of the evidence the } \\
\text { EPOS2020 steering group cannot advise on the use of nasal corticosteroids in children with acute } \\
\text { post-viral rhinosinusitis. }\end{array}$ \\
\hline Antihistamines & $1 b(-)$ & $\begin{array}{l}\text { There is one study evaluating antihistamines versus placebo in addition to (ineffective) antibiotics } \\
\text { in children with post-viral ARS showing no additive effect of antihistamines over the treatment } \\
\text { given. Based on the very low quality of the evidence, the EPOS2020 steering group cannot advise } \\
\text { on the use of antihistamines in post-viral ARS. }\end{array}$ \\
\hline Bacterial lysates & $\mathrm{lb}$ & One study has shown benefit in the use of OM-85-BV for shortening the duration of illness. \\
\hline
\end{tabular}

ARS, acute rhinosinusitis. 
Figure 1.4.1. Integrated care pathway of acute rhinosinusitis.

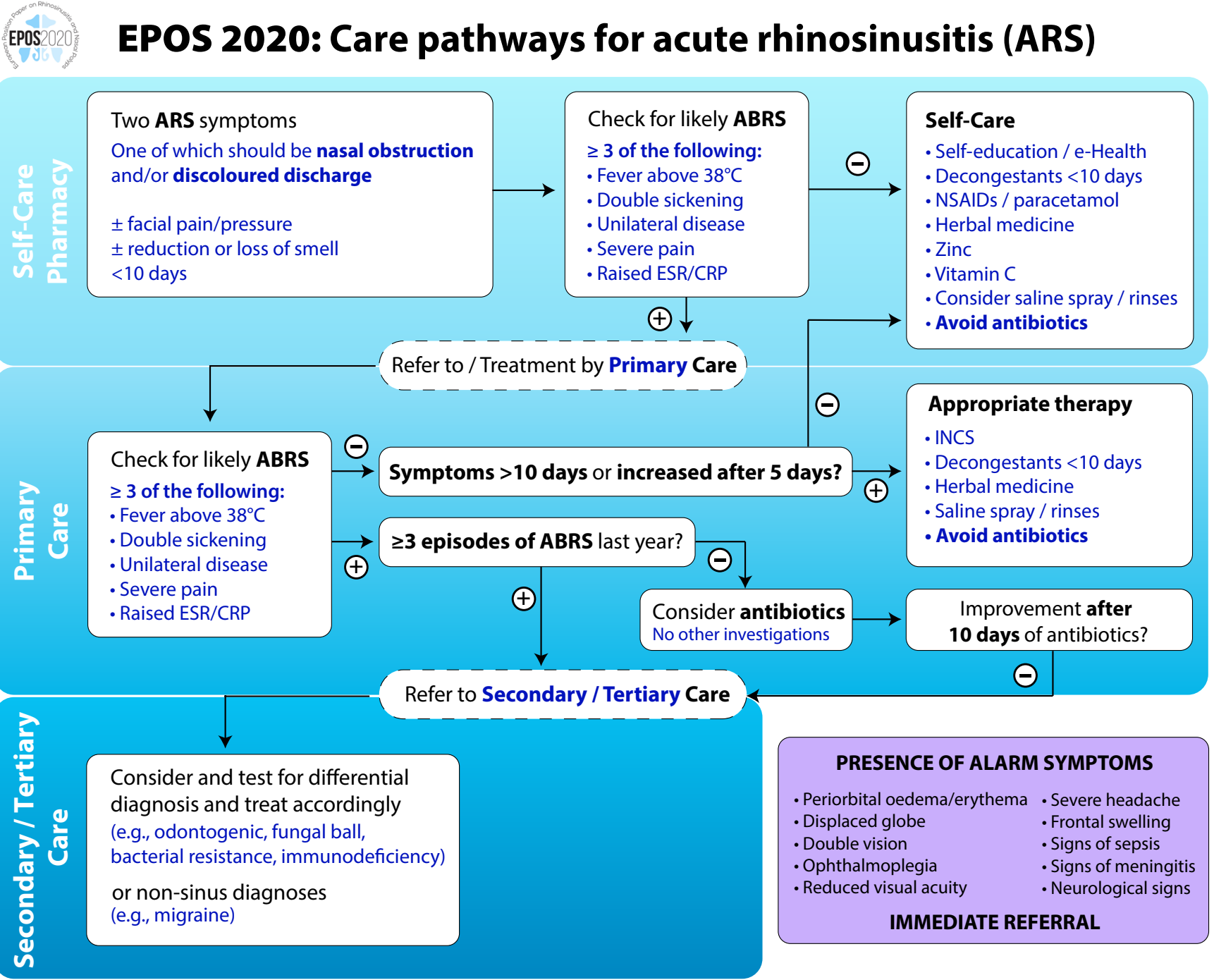

ABRS, acute bacterial rhinosinusitis; INCS, intranasal corticosteroids.

asthma, and hay fever ${ }^{(28)}$. In the USA, the direct costs for the management of CRS are now between $\$ 10$ and $\$ 13$ billion per year, or $\$ 2609$ per patient per year. In Europe, Wahid et al. reported 2974 GBP on costs for primary and secondary care extrapolated for a year period compared to 555 GBP in the control group and 304 versus 51 GBP out-of-pocket expenditure ${ }^{(29)}$. Lourijsen et al. found yearly direct costs of 1501 euro per year in a group of patients with $\mathrm{CRSWNP}^{(30)}$. Overall CRS leads to an incremental direct healthcare expenditure of 2500 euro per patient per year. The highest direct costs were associated with patients who had recurrent polyposis after surgery ${ }^{(31)}$. However, whilst surgery is expensive, varying from up to $\$ 11,000$ in USA to $\$ 1100$ in India ${ }^{(32-34)}$, it results in a decrease in direct costs in the subsequent two post-operative years ${ }^{(35)}$. The indirect costs of rhinosinusitis are much greater than the direct costs. Since $85 \%$ of patients with rhinosinusitis are of working age (range: 18-65 years old), indirect costs such as missed workdays (absenteeism) and decreased productivity at work (presenteeism) significantly add to the economic burden of the disease ${ }^{(35)}$. As a consequence, rhinosinusitis is one of the top 10 most costly health conditions to US employers ${ }^{(36)}$. Overall, the total indirect costs of CRS were estimated to be in excess of $\$ 20$ billion per year in the USA ${ }^{(37)}$ mainly due to presenteeism.

\subsection{Acute rhinosinusitis including common cold and recurrent ARS in adults and children}

Chapter 4 describes the epidemiology, pathophysiology, diagnosis and differential diagnosis, and management of ARS in adults and children. Also, a new integrated care pathway based on all the evidence is proposed.

\subsubsection{Epidemiology}

In EPOS2012 the division of ARS into viral ARS (common cold), post-viral ARS and ABRS (acute bacterial rhinosinusitis) was proposed. In the last decade studies have been performed using 
Table 1.4.4. Treatment evidence and recommendations for adults with acute bacterial rhinosinusitis (ABRS).

\begin{tabular}{|c|c|c|}
\hline Therapy & $\begin{array}{l}\text { Level of } \\
\text { evidence }\end{array}$ & GRADE recommendation \\
\hline Antibiotics & $1 \mathrm{a}$ & $\begin{array}{l}\text { Antibiotics are effective in a select group of patients with symptoms and signs suggestive of ABRS. From the limited } \\
\text { data available (two studies versus one) it seems that amoxicillin/penicillin (beta-lactams) especially are effective } \\
\text { and moxifloxacin (fluoroquinone) is not. The efficacy of beta-lactams is evident at day three where patients } \\
\text { already experience better symptom improvement and continues with a higher number of cures at completion of } \\
\text { treatment. However, careful patient selection for those with ABRS is needed to avoid unnecessary use of antibiotics } \\
\text { and side effects. }\end{array}$ \\
\hline Antihistamines & $1 \mathrm{~b}(-)$ & $\begin{array}{l}\text { There is one study evaluating antihistamines versus placebo in adults with allergic rhinitis and ABRS showing no } \\
\text { effect. Based on the very low quality of the evidence, the EPOS2020 steering group cannot advise on the use of } \\
\text { antihistamines in post-viral ARS and ABRS. }\end{array}$ \\
\hline $\begin{array}{l}\text { Nasal irrigation with } \\
\text { saline }\end{array}$ & $1 \mathrm{~b}(-)$ & $\begin{array}{l}\text { One study comparing hypertonic saline nasal spray, isotonic saline nasal spray and no treatment in addition to } \\
\text { antibiotics did not find a difference between the groups. Based on the very low quality of the evidence no advice } \\
\text { can be given about the use of nasal saline irrigation. }\end{array}$ \\
\hline Sodium Hyaluronate & $\mathrm{lb}$ & $\begin{array}{l}\text { One study evaluating sodium hyaluronate compared to placebo in a nebulizer ampoule for nasal douching in } \\
\text { addition to levofloxacin and prednisone showed significantly fewer symptoms and better smell threshold in the } \\
\text { sodium hyaluronate group. Based on the very low quality of the evidence no advice can be given about the use of } \\
\text { sodium hyaluronate. }\end{array}$ \\
\hline
\end{tabular}

ABRS, acute bacterial rhinosinusitis; ARS, acute rhinosinusitis.

Table 1.4.5. Treatment evidence and recommendations for children with acute bacterial rhinosinusitis (ABRS).

\begin{tabular}{|c|c|c|}
\hline Therapy & $\begin{array}{l}\text { Level of } \\
\text { evidence }\end{array}$ & GRADE recommendation \\
\hline Antibiotics & $1 \mathrm{a}(-)$ & $\begin{array}{l}\text { Data on the effect of antibiotics on the cure/improvement of symptoms in ABRS in children are very limited. There } \\
\text { are only two studies with limited numbers that do not show a significant difference over placebo but do show a } \\
\text { significant higher percentage of adverse events. Larger trials are needed to explain the difference between adults } \\
\text { where antibiotics in ABRS has been shown to be effective and this outcome. }\end{array}$ \\
\hline Mucolytics & $1 b(-)$ & Erdosteine as an adjunct to antibiotic was not more effective than placebo \\
\hline
\end{tabular}

ABRS, acute bacterial rhinosinusitis.

this classification. In a recent Dutch paper using the GA2LEN questionnaire a prevalence of $18 \%$ (17-21\%) was found for symptoms pointing to post-viral ARS in three different cities in the Netherlands ${ }^{(38)}$. ABRS is a rare disease with an incidence of $0.5-2 \%$ of viral ARS (common cold) ${ }^{(2,39)}$. RARS is defined as $\geq 4$ episodes per year with symptom free intervals ${ }^{(40-43)}$. Each episode must meet the criteria for acute post-viral (or bacterial) rhinosinusitis. The EPOS2020 steering group advises to have at least one proven diagnosis of post-viral ARS with endoscopy and/or CT scan before a diagnosis of RARS is considered.

\subsubsection{Predisposing factors for ARS and RARS}

Predisposing factors for ARS are seldom evaluated. There is some indication that anatomical abnormalities may predispose to recurrent acute rhinosinusitis (RARS) ${ }^{(44-47)}$. Active and passive smoking predisposes to ARS and there is some evidence that concomitant chronic disease may increase the chance of getting ARS following an influenza infection ${ }^{(48-50)}$.

Other potential factors like allergy and GORD do not seem to predispose to $\operatorname{ARS}^{(51,52)}$.

\subsubsection{Pathophysiology of ARS}

The pathophysiology of ARS is systematically evaluated, again trying to organize the literature based on the different categories of ARS. Since EPOS2012, there have been increasing experimental data supporting the fact that nasal epithelium is the primary portal of entry for respiratory viruses as well as an active component of initial host responses against viral infection. The cascade of inflammation initiated by nasal epithelial cells will lead to damage by the infiltrating cells, causing oedema, engorgement, fluid extravasation, mucus production and sinus obstruction in the process, eventually leading to ARS or exacerbating ARS (see chapter 4.2.).

\subsubsection{Diagnosis and differential diagnosis of ARS in adults and children}

Post-viral ARS is a common condition in the community, usually following viral URTI. Most acute viral URTI infections are selflimiting, thus post-viral ARS should not be diagnosed before 10 days' duration of symptoms unless there is a clear worsening of symptoms after five days.

Subjective assessment should take into account the severity and the duration of symptoms (see above). The recommended method of assessing severity of symptoms is with a visual analogue scale (VAS) recorded by the patient on a $10 \mathrm{~cm}$ line giving a score on a measurable continuum of 1 to 10 .

Bacterial infection may occur in ARS, but in most cases antibiotics have little effect on the course of the illness (see 1.4.5.). 
A number of studies have attempted to provide clinicians with combinations of symptoms and signs predicting more severe disease, particularly of a bacterial infection and the likelihood of a response to antibiotics ${ }^{(53)}$. The EPOS2020 steering group decided to maintain suggestions made in the earlier EPOS versions: at least three of five symptoms of discoloured discharge, severe local pain, fever, elevated ESR/CRP and double sickening.

\subsubsection{Treatment of ARS in adults and children}

For EPOS2020 a systematic review was performed evaluating treatment of the different categories of ARS (viral, post-viral or ABRS) separately. For acute viral rhinosinusitis we found many excellent systematic reviews and report on them. For post-viral rhinosinusitis and ABRS a systematic review of the literature has been performed for children and adults. The different treatments, levels of evidence and GRADE recommendations are reported in Tables 1.4.1-1.4.5. For medication not mentioned in these tables, we could not find RCTs.

Based on the systematic review, a new integrated care pathway is proposed (Figure 1.4.1.). In this figure it is emphasized that the treatment of almost all patients with ARS should be symptomatic, if needed, combined with local corticosteroids. The place for antibiotics is very limited and they should only be given in situations pointing to severe disease with symptoms and signs such as high fever, double sickening, severe pain and elevated $\mathrm{ESR}^{(3)}$.

Finally, in chapter 4 the complications of ABRS are discussed. Complications of bacterial rhinosinusitis are rare but potentially serious. However, a number of studies have shown that they are not prevented by routine prescribing of antibiotics. A low threshold of suspicion must always be maintained for their early diagnosis.

\subsection{Epidemiology, predisposing factors, pathophysiology, and diagnosis of CRS}

\subsubsection{Epidemiology and predisposing factors}

The overall prevalence of symptom-based CRS in the population has been found to be between $5.5 \%$ and $28 \%(4,5,54,55)$, CRS is more common in smokers than in non-smokers ${ }^{(4)}$. The prevalence of self-reported physician-diagnosed CRS is highly correlated with the prevalence of EPOS-diagnosed CRS ${ }^{(4)}$. When symptoms are combined with endoscopy or CT scan prevalence is reduced to $3-6 \%{ }^{(56-58)}$.

CRS is associated with asthma, with a prevalence of asthma around $25 \%$ in patients with CRS compared to $5 \%$ in the general population. CRS is also associated with COPD, N-ERD, hypogammaglobulinemia, and GORD (see chapter 5.1). Smoking, air-pollution and occupational exposure are negatively correlated with CRS (symptoms).

The prevalence of allergy in CRS may vary by phenotype, with CCAD and AFRS having a stronger association than CRSwNP and CRSsNP( $(59,60)$. An important percentage of subjects diagnosed with chronic upper airway disease report alcohol-induced worsening of their symptoms ${ }^{(61)}$.

\subsubsection{Genetics}

The current knowledge base on the genomics of CRS disease offers the promise of identifying new mechanisms of disease development and markers predicting optimal response to available therapies. However, for the moment, genetics do not allow prediction of disease or outcome and its uses are currently restricted to extreme cases to understand the molecular underpinning of pathologies. It is probable that over the coming years we will identify individual or complex genetic traits conferring susceptibility to CRS, evolution of disease, and response to medical or surgical treatment ${ }^{(62,63)}$.

Figure 1.5.1. Aetiology and pathogenesis of CRS.

EPOS2020 Etiology and Pathogenesis of CRS

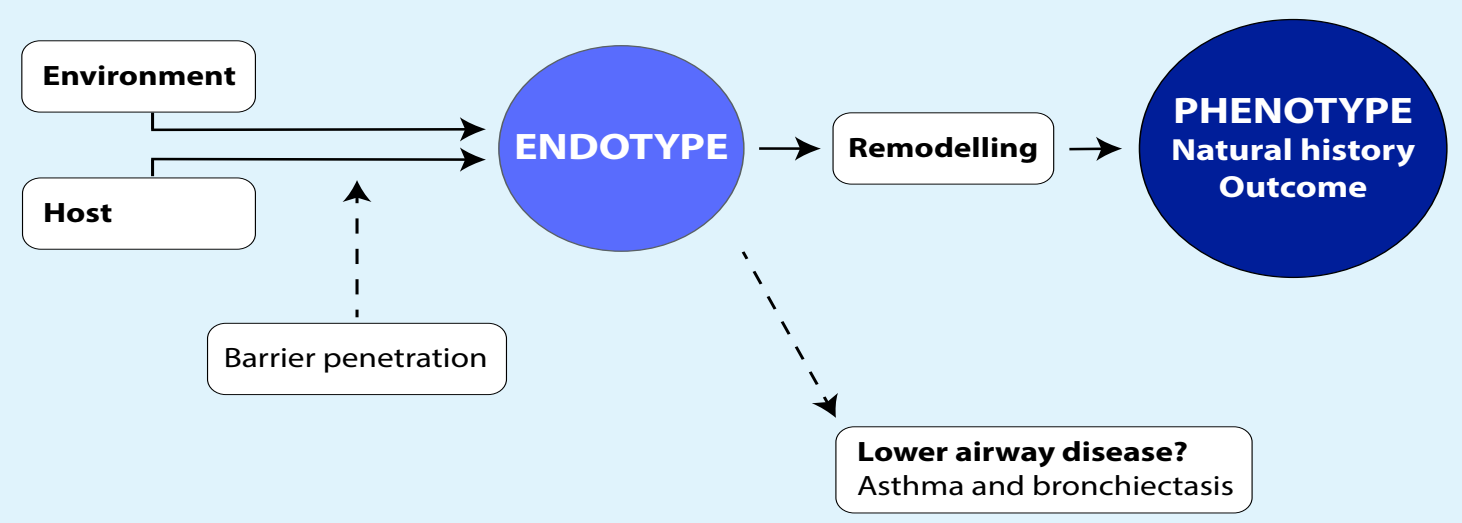

CRS, chronic rhinosinusitis 


\subsubsection{The emerging clinical relevance of CRS pathophysiology}

Research into the aetiology and pathogenesis of chronic rhinosinusitis has been largely irrelevant to the clinician, with minimal impact on management. Historically, CRS has been divided into two groups based on the presence or absence of polyps and, in rough overview, corticosteroids were commonly used for CRSwNP and antibiotics for CRSsNP. The rationale for these regimens was based on decades-old presumptions that CRSsNP was the result of an incompletely treated acute bacterial infection that then became 'chronic' and CRSwNP had some relationship to local or systemic 'allergy'. Surgery was the only option for failures. It has been clear for at least 20 years that this assessment was simplistic at best. The emerging view was that CRS was a syndrome with a multifactorial aetiology resulting from a dysfunctional interaction between various environmental factors and the host immune system. It was, however, very unclear which environmental and host factors were important even in the population at large, let alone in an individual CRS patient. Nevertheless, research was undertaken with the initial goal of examining causation of CRS as a route to therapy. Later, the results of these efforts shifted emphasis toward the tissue effects generated by those causative factors and away from the factors themselves. The following brief synopsis describes how that 20-year journey is finally beginning to impact how we treat patients with CRS.

Research into the aetiology and pathogenesis of CRS was first energized by the work on fungus, which was proposed as the key aetiologic agent, at least in patients with recalcitrant CRS. This was followed shortly after with Staphylococcus aureus being proposed as a rival pathogen, perhaps in biofilm format to enable greater resistance. Later, the more general hypothesis of microbial dysbiosis was proposed, wherein the collective microbial community was abnormal and pathogenic, propagating sinonasal inflammation occurred at anatomically vulnerable sites. Unfortunately, therapies directed at fungi, staphyloccus aureus and even the microbiome as a whole have been, at best, underwhelming. This suggested the opposite therapeutic tactic: shift attention away from antimicrobials and towards the goal of correcting any immune dysfunction in the individual CRS patient. By then it was understood that both the nose and sinuses were not sterile: a process which begins at birth with the rapid colonization by viruses, bacteria and fungi. In healthy individuals, the mucosa serves as a relative barrier modulating interaction with the host immune system, promoting tolerance and symbiosis as well as preventing or limiting inflammation. In patients with CRS, the barrier is penetrated with resultant chronic inflammation leading to, in many cases, tissue remodeling and clinical symptoms. In theory, identification of specific genetic or epigenetic variations in the host immune system that permit CRS to develop should be possible, providing targets for future therapies. Unfortunately, outside of cystic fibrosis and CFTR, the genetics of CRS appear to be quite complex for the typical patient, involving multiple genes, each with a small effect size. Moreover, genetic studies on the large populations necessary to identify these genes would be very expensive and have generally not been undertaken. Effectively, this approach was rendered impractical and therapeutic approaches to manage CRS based on putative aetiologies - either host or environment based - have made relatively little clinical impact. Nevertheless, this entire body of work revealed a great deal about the nature of the inflammation present in the tissue of CRS patients.

The failure of aetiology-based treatments for CRS is, in retrospect, not surprising since CRS is typically an adult onset disorder with diagnosis most commonly in the fifth decade of life. This extended premorbid time course suggests a complex host-environmental interaction, with great variability in nature, sequence and intensity of exogenous stressors including superimposed stochastic events. Dissecting out the process in an individual patient would be a daunting, if not impossible task that might still not lead to any therapeutic path forward. By analogy, identifying smoking as carcinogenic may help prevent future cancers through avoidance, but it will not significantly affect treatment recommendations for a patient who has already acquired the problem. The accompanying line drawing (Figure 1.5.1.) illustrates a contemporary model of CRS pathogenesis. Rather than analysis of the complex and usually unknown factors that cause CRS in an individual patient, interest now is centred on the resulting inflammation that develops in the sinus tissue. The focus is toward the identification of the molecular pathway(s) or endotypes that have been activated. This effort has been aided by recent advances in our understanding of the physiologic immune response against pathogens across mucosal barriers. When the barrier is breached, a self-limited immunodefensive response is generated, characterized by a cellular and cytokine repertoire targeting one of the three classes of pathogens: type 1 immune responses target viruses; type 2 responses target parasites and type 3 target extracellular bacteria and fungi, all of which resolve with elimination of the pathogens and restoration of barrier integrity. In cases of CRS, barrier penetration results in a chronic inflammatory response that fails to resolve, but still typically utilizes the type 1, 2 or 3 pathways alone, or in combinations. Type 2 inflammation is characterized by cytokines IL-4, IL-5 and IL-13 as well as activation and recruitment of eosinophils and mast cells. CRS research has revealed that patients with a pure or mixed type 2 endotype tend to be much more resistant to current therapies, exhibiting a high recurrence rate when compared with pure type 1 or 3 endotypes. Furthermore, while type 2 CRS clearly varies between patients by intensity of inflammation, subtypes may exist wherein discrete aspects of the pathway are relatively enhanced (e.g. mast cell activation, eosinophil activation, and plasma cells activity). Most importantly, biologic agents have now become available that target specific aspects of type 2 inflammation. In the very near future, it may be possible to offer personalized medicine for CRS patients where treatment is based on molecular biomarkers for the endotype or subendotype activated in an individual patient.

Remodelling of sinonasal tissues in CRS consists most prominently of polyp formation, goblet cell hyperplasia and epithelial barrier abnormalities, which in aggregate, may account for many or most of the CRS symptoms. In the case of the barrier remodelling, the result is greater permeability, likely facilitating persistence or recurrence of CRS. All of these changes are most apparent in type 2 CRS, possibly accounting 
Figure 1.6.1. Treatment evidence and recommendations for adults with chronic rhinosinusitis.

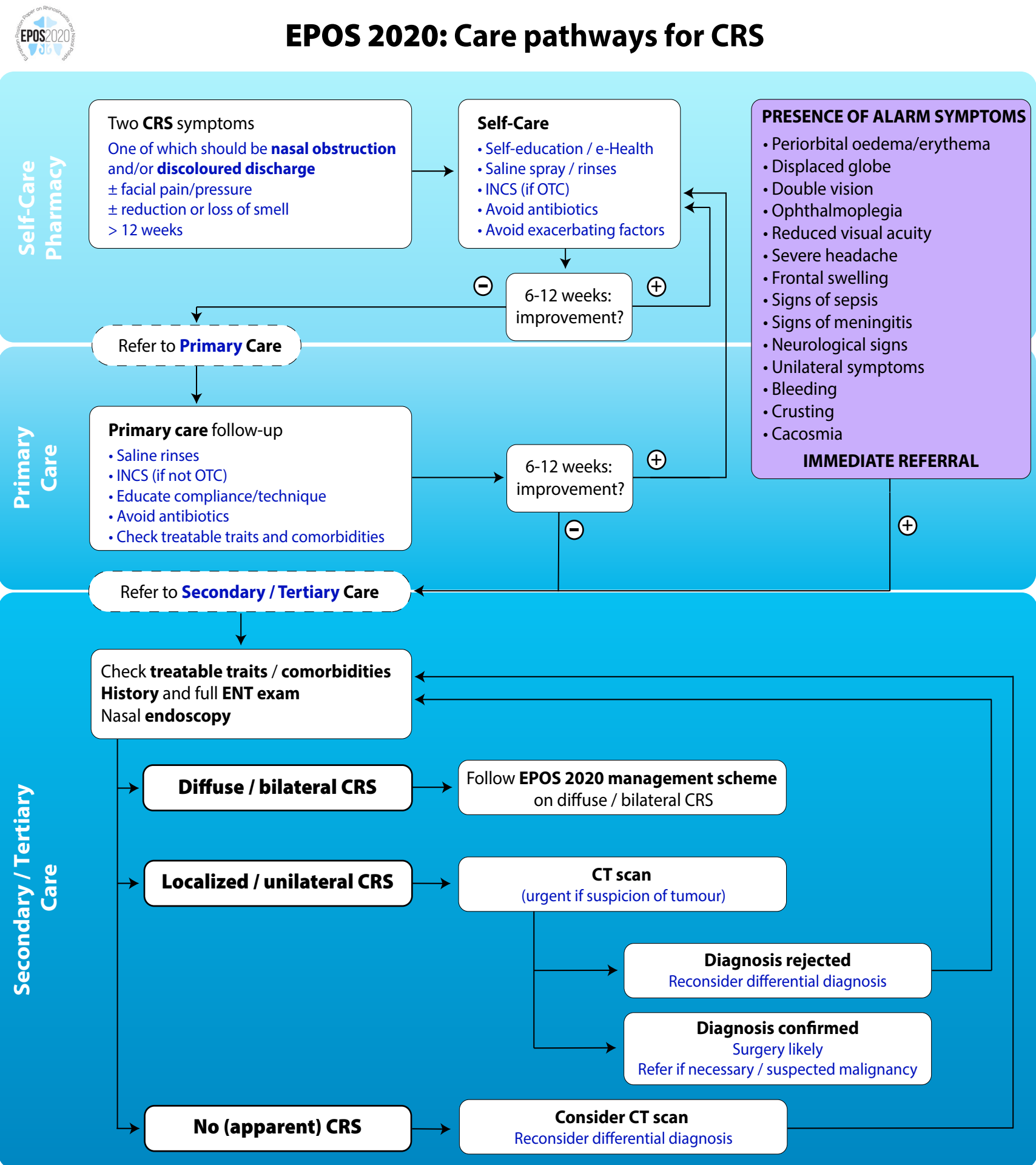

CRS: chronic rhinosinusitis; CT, computed tomography; INCS, intranasal corticosteroids spray; OTC, over-the-counter.

for the observed greater symptomatology and higher rate of treatment failure. The precise relationship between the endotype and the remodelling pattern is not completely clear but recent evidence suggests that it may be cause and effect as depicted in Figure 1.5.1. Specifically, the use of biologic agents that suppress the type 2 endotype, also shrink polyps. Reversal of goblet cell hyperplasia has not yet been documented, but in vitro studies suggest that barrier-related remodelling is driven directly, in large measure, by canonical type 2 cytokines. Biologic agents that suppress type 2 inflammation may, therefore, suppress the inflammation, reverse the remodelling and limit recurrence, thereby altering the clinical course of the most severe CRS phenotypes. Further research into type 2 inflammation will be extremely helpful in the use of these 
Figure 1.6.2. EPOS2020 management scheme on diffuse CRS.

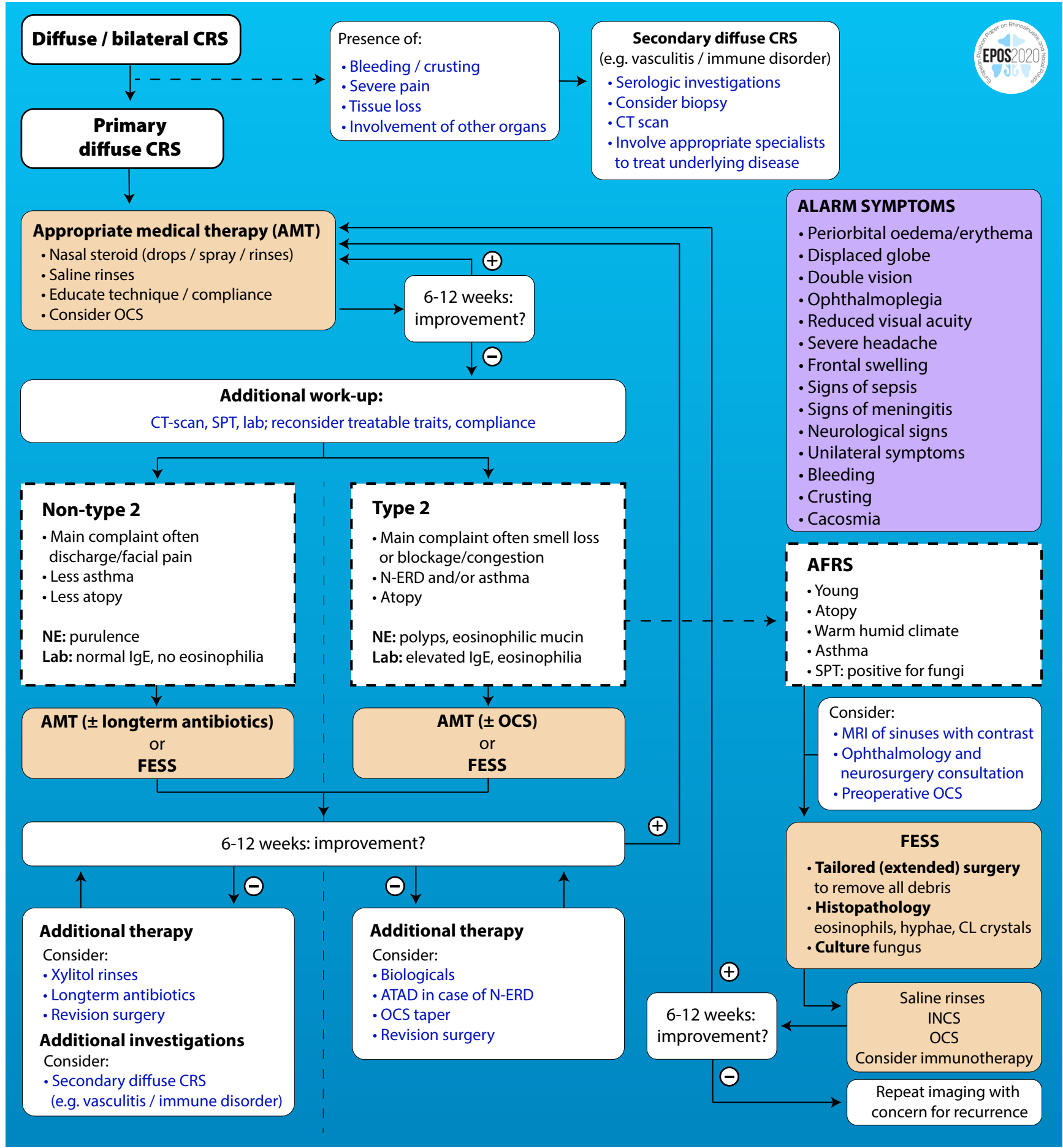

For explanation of (primary and secondary) diffuse CRS see 1.2.3.

AMT, appropriate medical therapy; ATAD, Aspirin treatment after desensitisation; CRS, chronic rhinosinusitis; CT, computed tomography; FESS, functional endoscopic sinus surgery; INCS, intranasal corticosteroid spray; MRI, magnetic resonance imaging; NE, nasal endoscopy; N-ERD, NSAID-exacerbated respiratory disease; OCS, Oral corticosteroids; SPT, Skin prick test. 
powerful drugs, which have the potential to revolutionize CRS treatment ${ }^{(64)}$.

\subsubsection{Differential diagnosis and diagnostic tools}

\subsubsection{Differential diagnosis}

It was decided to include more information in EPOS2020 to better allow differential diagnosis of rhinosinusitis from certain other conditions and common symptoms, notably allergic and non-allergic rhinitis, olfactory loss and facial pain. We also include an updated and expanded range of diagnostic tools, though many have not substantially changed since 2012 . Upper airway diseases present with a variable pattern of common symptoms such as nasal obstruction and discharge, making the epidemiological diagnosis of CRS difficult to differentiate from allergic and nonallergic rhinitis based on symptomatic grounds. Combining data from different studies leads to a picture of significant overlap in prevalence and severity of symptomatology. However, as there are generally less inflammatory changes seen on CT sinuses in AR and NAR than $\mathrm{CRS}^{(65)}$ a combination of symptoms, CT scan and nasal endoscopy can point in the right direction.

Olfactory loss is one of the cardinal symptoms of CRS but has a wide differential diagnosis ${ }^{(66)}$. The prevalence of olfactory disorders in the general population is estimated to be 3-5 \% for total smell loss (anosmia) and 15-25\% for partial impairment (hyposmia) ${ }^{(67,68)}$. In CRS the mechanism leading to olfactory impairment is twofold: inflammatory and purely mechanical due to obstruction of the olfactory cleft ${ }^{(69,70)}$, which explains why not all patients have an olfactory benefit from surgical removal of polyps alone but also require subsequent anti-inflammatory treatment. However, CRS-related olfactory loss has a good success rate of improvement if the CRS is treated even if not always sustained in the long-term.

Facial pain is another cardinal symptom of CRS which can occur in many other conditions ${ }^{(71)}$. However, facial pain when it occurs alone is rarely caused by CRS and, therefore, when it occurs without other nasal complaints or abnormalities on examination, it should not (primarily) be addressed surgically.

\subsubsection{Diagnostic tools}

The different imaging modalities in diagnosing rhinosinusitis [conventional X-ray, computerized tomography (CT), cone beam CT and magnetic resonance imaging (MRI)] have been evaluated $^{(72)}$. Overall CT scan remains the gold standard in the radiologic evaluation of rhinologic disease, notably $\mathrm{CRS}^{(73-75)}$. However, in acute rhinosinusitis, the diagnosis is made on clinical grounds and CT is not recommended ${ }^{(3)}$ unless the condition persists despite treatment, or a complication is suspected ${ }^{(76)}$. Conventional sinus $\mathrm{X}$-rays are no longer indicated in either ARS or CRS.

The most commonly used and validated scoring system of sinonasal inflammatory change remains the Lund-Mackay score (LMS) which gives a maximum score of 24 or $12 /$ side $^{(77)}$. An LMS of 2 or less has an excellent negative predictive value, and an LMS of 5 or more has an excellent positive predictive value, strongly indicating true disease. In CRS, CT was not normally recommended until after an appropriate course of medical therapy had failed ${ }^{(3,78)}$ and without an intervening acute episode but more recent studies suggest that early CT scanning may be more cost-effective as compared to extended courses of antibiotics given empirically and is preferred by patients ${ }^{(79-81)}$.Multi-detector CT (MDCT) scanners and conebeam $C T$ are reducing the radiation dose whilst preserving image quality by shortening the scan time and using postprocessing techniques ${ }^{(82,83)}$ without compromising anatomical accuracy ${ }^{(84)}$, making them increasingly attractive ${ }^{(85,86)}$.

In the measurement of health-related quality-of-life (HRQL), a wide range of validated patient reporting outcome measures (PROMS) are available but currently none of the established PROMS capture all the desired aspects of CRS; the SNOT-22 fails to capture disease duration or medication usage. Current recommendations include the use of SNOT-22 scores repeated over time, Lund Kennedy endoscopic scores, and additional questions to evaluate the need for systemic medications or progression to surgery, compliance with and side effects of treatment, additional information on symptom frequency, and impact on ability to perform normal activities ${ }^{(87)}$.

Nasal endoscopy remains an essential part of the rhinological examination. A recent systematic review analysed the accuracy of nasal endoscopy in diagnosing chronic rhinosinusitis (CRS) compared with paranasal sinus computed tomography (CT). Sixteen observational or retrospective studies were included resulting in a high correlation $(r=0.85 ; 95 \%$ confidence interval [Cl][0.78-0.94], $\mathrm{p}<0.0001, \mathrm{I}^{2} 77 \%$ ) between endoscopy and CT in terms of the diagnostic accuracy for $\mathrm{CRS}^{(88)}$.

A clinical history supported with a skin prick test or serum IgE measuremernt will probably remain the gold standard of the upper airway allergy diagnosis but advances are expected from the molecular in vitro diagnosis which may change this trend, due to improved technology which enables faster diagnosis on a broader panel of allergens ${ }^{(89,90)}$.

As CRS patients are commonly not fully aware of their olfactory impairment, or are unable to estimate the severity of the loss, the use of smell tests is recommended in order to objectively evaluate this disorder ${ }^{(91,92)}$. The most widely used remain the North American UPSIT ${ }^{(93)}$, its short version (SIT, B-SIT) and the European Sniffin'Sticks ${ }^{(94)}$. Although there are many others, all have cultural bias and there have been recent advances to overcome this with culturally unbiased, universally usable smell tests ${ }^{(95)}$.

Nasal obstruction is the most significant of the cardinal symptoms of rhinosinusitis and nasal patency may be objectively evaluated with peak nasal inspiratory flow (PNIF), (active anterior) rhinomanometry (AAR), and acoustic rhinometry (AR) Newer methods such as computational fluid dynamics ${ }^{(96)}$ are presently mainly used for research purposes ${ }^{(97,98)}$ but may be of value in the future.

In addition to confirming diagnosis, histopathology is becoming more important to assist in endotyping of inflammatory disease, thereby directing potential therapies, e.g. biologics. Eosinophilic CRS (eCRS) requires quantification of the numbers of eosinophils, i.e. number/high powered field (HPF (400x) and EPOS2020 supports 10 or >/HPF. Further stratification may be 
made between those with10-100 eosinophils per HPF in two or more areas and those with $>100$ eosinophils per HPF in two or more areas ${ }^{(99)}$. The amount of eosinophilic infiltration and the overall intensity of the inflammatory response are closely related to the prognosis and severity of disease ${ }^{(100)}$. Until recently most blood tests in patients with CRS were performed to diagnose immunodeficiencies and vasculitis. However, recently the options to treat with biologicals has put more emphasis on markers of type 2 disease, although as it stands we are not aware of biomarkers that can predict response to biologicals in $\mathrm{CRS}^{(101)}$. For microbiology, in addition to the standard culture-dependant tests, newer culture-independent techniques including next generation sequencing may provide significant insight into CRS pathophysiology. This could include sequencing of all DNA (metagenomics) or all transcribed RNA (metatranscriptomics) or identification of proteins (metaproteomics) or metabolites (metabolomics), showing not only the true diversity and structure, but also the full genetic potential and in situ activity of the mucosa-associated microbiota ${ }^{(102)}$.

EPOS2020 also includes an update on mucociliary testing and other tests for primary ciliary dyskinesia (PCD), sweat testing and other tests for cystic fibrosis and advances in genetic testing as well as new diagnostic tools for N-ERD. Finally, the lower respiratory tract is not forgotten and the full range of available investigations are covered from peak expiratory flow to provocation tests and expired nitric oxide measurement.

\subsection{Management of chronic rhinosinusitis in adults}

\subsubsection{Introduction}

An important difference compared to EPOS2012 is that we have decided to move away from differentiating between the management of CRSsNP and CRSwNP per se. The understanding of the last decade of endotyping of CRS and the consequences of endotypes for the management of disease has led to the decision to describe management of CRS based on endotyping and phenotyping.

We propose a new clinical classification based on the disease being localized (often unilateral) or diffuse (always bilateral). Both these groups can be further divided into type 2 or nontype 2 disease (Figure 1.2.1.). The major challenge is to find reliable biomarkers that define type 2 inflammation and predict reaction to medication. Unfortunately, recent large studies with monoclonal antibodies directed at type 2 endotypes have not found reliable biomarkers to predict response to treatment ${ }^{(103,104)}$. For the moment the combination of phenotype (e.g. CRSWNP, N-ERD), response to treatment (systemic corticosteroids) and possibly also markers like eosinophils, periostin and IgE either in blood or tissue lead us to the best estimation of the endotype and reaction to treatment. This is a rapidly evolving field at the moment and we expect that frequent updates will be necessary.

\subsubsection{Management of CRS: an integrated care pathway}

For the management of CRS, a full systematic review of the literature has been performed (see chapter 6 and Table 1.6.1.). Many forms of localised CRS (Figure 1.2.1.) in general, either type
2 or non-type 2 , are not responsive to medical treatment and need surgery. For that reason, we advise patients with unilateral disease to be referred to secondary care for further diagnosis. Many studies do not make a clear differentiation between CRSsNP and CRSwNP. Very few studies further define CRS phenotypes or endotypes in the disease. CRS research has revealed that patients with a pure or mixed type 2 endotype tend to be more resistant to current therapies, exhibiting a high recurrence rate when compared with pure type 1 or 3 endotypes.

For diffuse, bilateral CRS, local corticosteroids and saline remain the mainstay of the treatment (Figure 1.6.1.).

Furthermore, the integrated care pathway (ICP) advises to check treatable traits, to avoid exacerbating factors and advises against the use of antibiotics. In secondary care, nasal endoscopy can confirm disease, point to secondary CRS (e.g. vasculitis) and further differentiate between localized and diffuse disease (Figure 1.6.2.).

In addition, emphasis is put on optimum techniques of medication delivery and compliance. If treatment with nasal steroid and saline is insufficient, an additional work-up with CT scan and endotyping is relevant. Depending on the endotype indication, treatment can be tailored to a more type 2 or nontype 2 profile. International guidelines differ regarding whether long-term antibiotics and oral steroids should be included as part of adequate medical therapy (AMT), reflecting conflicting evidence in the current literature ${ }^{(3,78,105)}$, and concerns with regard to side-effects. There is a lot of debate on the appropriate moment for surgery for $\mathrm{CRS}^{(105)}$. In a recent study for adult patients with uncomplicated CRS, it was agreed that ESS could be appropriately offered when the CT Lund-Mackay score was $\geq 1$ and there had been a minimum trial of at least eight weeks' duration of a topical intranasal corticosteroid plus a short-course of systemic corticosteroid (CRSwNP) or either a short-course of a broad spectrum / culture-directed systemic antibiotic or the use of a prolonged course of systemic low-dose anti-inflammatory antibiotic (CRSsNP) with a post-treatment total SNOT-22 score $\geq 20$. These criteria were considered the minimal threshold, and clearly not all patients who meet the criteria should have surgery, but their application should reduce unnecessary surgery and practice variation. A subsequent study applied these criteria retrospectively to patients recruited to a multicentre cohort study and found that patients where surgery was deemed 'inappropriate' reported significantly less improvement in their quality of life postoperatively ${ }^{(106)}$.

It is important to emphasize that CRS is a chronic disease and ESS a step in the management that is primarily aimed at creating better conditions for local treatment. After surgery continuous appropriate medical treatment is mandatory. If surgery in combination with appropriate medical treatment fails, additional therapy can be considered. Options are the use of aspirin treatment after aspirin desensitisation (ATAD) ${ }^{(107)}$, longer (tapering) treatment with OCS, long term antibiotics ${ }^{(108)}$ and/or biologicals when indicated. 
Figure 1.6.3. Indications for biological treatment in CRS.

\section{Indications for biological treatment in CRSwNP}

\section{Presence of bilateral polyps in a patient who had ESS*}

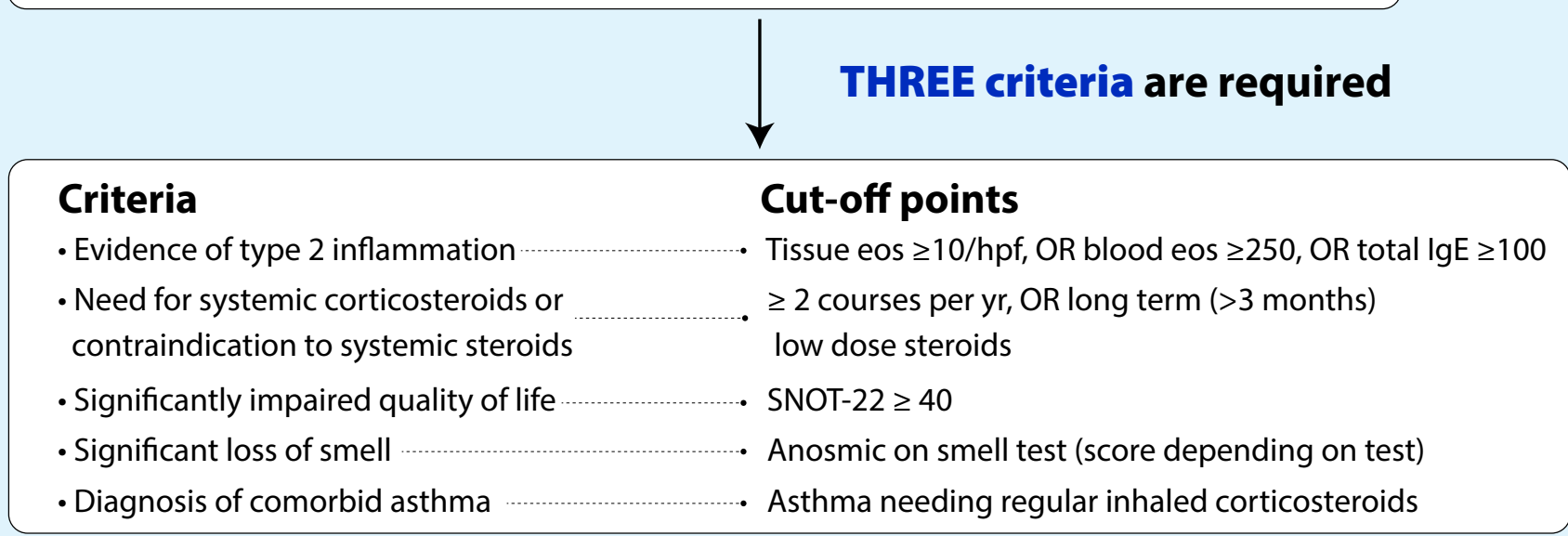

*exceptional circumstances excluded (e.g., not fit for surgery)

CRS, chronic rhinosinusitis; CRSwNP: chronic rhinosinusitis with nasal polyps; ESS, endoscopic sinus surgery; hpf: high power field ( $x 400)$; SNOT-22, sino-nasal outcome test-22.

Figure 1.6.4. Response criteria for biologicals in the treatment of CRS.

\section{Defining response to biological treatment in CRSwNP}

\section{Evaluation of 5 criteria}

- Reduced nasal polyp size

- Reduced need for systemic corticosteroids

- Improved quality of life

- Improved sense of smell

- Reduced impact of co-morbidities
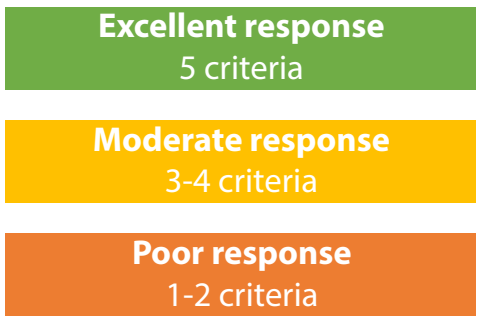

No response 0 criteria

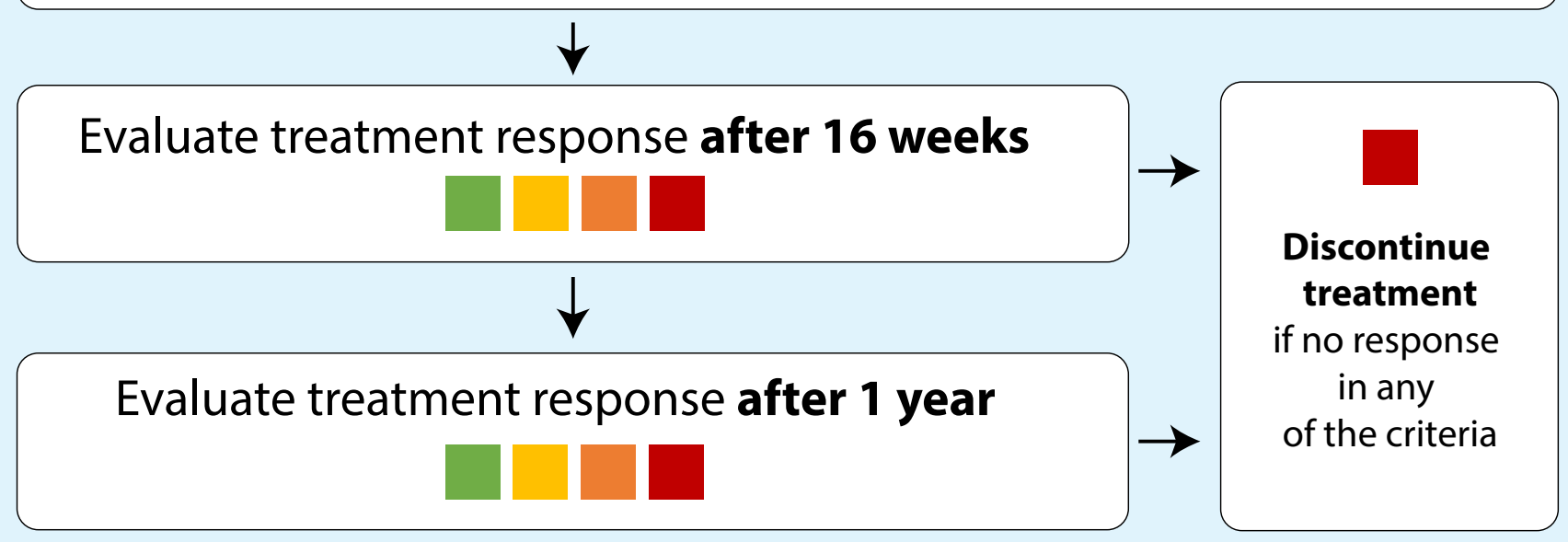


Table 1.6.1. Treatment evidence and recommendations for adults with chronic rhinosinusitis.

\begin{tabular}{|c|c|c|}
\hline Therapy & $\begin{array}{l}\text { Level of } \\
\text { evidence }\end{array}$ & GRADE recommendation \\
\hline Short term antibiotics for CRS & $1 \mathrm{~b}(-)$ & $\begin{array}{l}\text { There are only two small placebo-controlled studies, one in CRS and one in acute exacerbation of CRS. Both show no } \\
\text { effect on symptomatology apart from significantly reduced postnasal drip symptom scores at week } 2 \text { in the CRS study. } \\
\text { Seven studies evaluated two different antibiotics regimes, of which only one was placebo-controlled. One out of seven } \\
\text { studies in patients with CRS showed a significant effect on SNOT at } 2 \text { and } 4 \text { weeks and also one study a significant } \\
\text { improvment in symptoms of infection at day } 3 \text { to } 5 \text { in one antibiotic versus another in a mixed group of patients with } \\
\text { CRS and with acute exacerbation. The other } 5 \text { studies showed no difference in symptomatology. Only two of these seven } \\
\text { studies, both of which were negative, evaluated the effect after one month. } \\
\text { The EPOS2020 steering group, is uncertain, due to the very low quality of the evidence, whether or not the use of a short } \\
\text { course of antibiotics has an impact on patient outcomes in adults with CRS compared with placebo. Also, due to the very } \\
\text { low quality of the evidence, it is uncertain whether or not the use of a short course of antibiotics has an impact on patient } \\
\text { outcomes in adults with acute exacerbations of CRS compared with placebo. Gastrointestinal-related adverse events } \\
\text { (diarrhoea and anorexia) are frequently reported. }\end{array}$ \\
\hline $\begin{array}{l}\text { Short term antibiotics for acute } \\
\text { exacerbation of CRS }\end{array}$ & $1 \mathrm{~b}(-)$ & $\begin{array}{l}\text { The EPOS2020 steering group, is uncertain, due to the very low quality of the evidence, whether or not the use of a } \\
\text { short course of antibiotics has an impact on patient outcomes in adults with acute exacerbations of CRS compared with } \\
\text { placebo. Gastrointestinal-related adverse events (diarrhoea and anorexia) are frequently reported. }\end{array}$ \\
\hline Longterm antibiotics for CRS & $1 \mathrm{a}(-)$ & $\begin{array}{l}\text { The EPOS2020 steering group, due to the low quality of the evidence, is uncertain whether or not the use of long-term } \\
\text { antibiotics has an impact on patient outcomes in adults with CRS, particularly in the light of potentially increased risks of } \\
\text { cardiovascular events for some macrolides. Further studies with larger population sizes are needed and are underway. }\end{array}$ \\
\hline Topical antibiotics & $1 \mathrm{~b}(-)$ & $\begin{array}{l}\text { Topical antibacterial therapy does not seem to be more effective than placebo in improving symptoms in patients } \\
\text { with CRS. However, it may give a clinically non-relevant improvement in symptoms, SNOT- } 22 \text { and LK endoscopic score } \\
\text { compared to oral antibiotics. The EPOS } 2020 \text { steering group, due to the very low quality of the evidence, is uncertain } \\
\text { whether or not the use of topical antibiotic therapy has an impact on patient outcomes in adults with CRS compared with } \\
\text { placebo. }\end{array}$ \\
\hline Nasal corticosteroids & $1 \mathrm{a}$ & $\begin{array}{l}\text { There is high-quality evidence that long term use of nasal corticosteroids is effective and safe for treating patients with } \\
\text { CRS. They have impact on nasal symptoms and quality of life improvement, although the effect on SNOT-22 is smaller } \\
\text { than the minimal clinically important difference. The effect size on symptomatology is larger in CRSwNP (SMD -0.93, } \\
95 \% \mathrm{CI}-1.43 \text { to -0.44) than in CRSsNP (SMD -0.30, } 95 \% \mathrm{Cl}-0.46 \text { ). The meta-analysis did not show differences between } \\
\text { different kinds of nasal corticosteroids. Although in meta-analysis higher dosages and some different delivery methods } \\
\text { seem to have a larger effect size on symptomatology, direct comparisons are mostly missing. For CRSwNP, nasal } \\
\text { corticosteroids reduce nasal polyp size. When administered after endoscopic sinus surgery, nasal corticosteroids prevent } \\
\text { polyp recurrence. Nasal corticosteroids are well tolerated. Most adverse events reported are mild to moderate in severity. } \\
\text { Nasal corticosteroids do not affect intraocular pressure or lens opacity. The EPOS2020 steering group advises to use nasal } \\
\text { corticosteroids in patients with CRS. Based on the low to very low quality of the evidence for higher dosages or different } \\
\text { delivery methods and the paucity of direct comparisons the steering committee cannot advise in favour of higher } \\
\text { dosages or certain delivery methods. }\end{array}$ \\
\hline
\end{tabular}

Corticosteroid-eluting implants 1a The placement of corticosteroid-eluting sinus implants in the ethmoid of patients with recurrent polyposis after sinus surgery has a significant but small ( 0.3 on a 0-3 scale) impact on nasal obstruction but significantly reduces the need for surgery and reduces nasal polyp score. Based on the moderate to high quality of the evidence the steering group considered the use of corticosteroid-eluting sinus implants in the ethmoid an option.

Systemic corticosteroids $\quad$ A short course of systemic corticosteroid, with or without local corticosteroid treatment results in a significant reduction
in total symptom score and nasal polyp score. Although the effect on the nasal polyp score remains significant up to
three months after the start of treatment by that time there is no longer an effect on the symptom score. The EPOS2020
steering group felt that 1-2 courses of systemic corticosteroids per year can be a useful addition to nasal corticosteroid
treatment in patients with partially or uncontrolled disease. A short course of systemic corticosteroid postoperatively does
not seem to have an effect on quality of life. Systemic corticosteroids can have significant side effects.

\begin{abstract}
Antihistamines lb There is one study reporting on the effect of antihistamines in partly allergic patients with CRSwNP. Although there was no difference in total symptom score, the days with a symptom score $\leq 1$ was higher in the treated group. The quality of the evidence comparing antihistamines with placebo was very low. There is insufficient evidence to decide on the effect of the regular use of antihistamines in the treatment of patients with CRS.
\end{abstract}

Based on the very low quality of the available evidence, the EPOS2020 steering group is unsure about the potential use of montelukast in CRS and does not recommend its use unless in situations where patients do not tolerate nasal corticosteroids. Also, the quality of the evidence comparing montelukast with nasal corticosteroid is low. Based on the evidence, the steering group does not advise adding montelukast to nasal corticosteroid but studies evaluating the effect of montelukast in patients that failed nasal corticosteroids are missing. than MFNS alone without inducing rebound swelling. There was no effect of xylometazoline compared to saline in the early postoperative period. This review found a low level of certainty that adding a nasal decongestant to intranasal corticosteroids improves symptomatology in CRS. Although the risk of rebound swelling was not shown in this study, the EPOS2020 steering group suggests in general not to use nasal decongestants in CRS. In situations where the nose is very blocked, the temporary addition of a nasal decongestant to nasal corticosteroid treatment can be considered. 
Table 1.6.1. Cont.

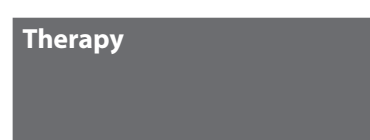

Nasal irrigation with saline
Level of evidence

\section{GRADE recommendation}

There are a large number of trials evaluating the efficacy of nasal irrigation. However, the quality of the studies is not always very good which makes it difficult to give a strong recommendation. However, the data show: Nasal irrigation with isotonic saline or Ringer's lactate has efficacy in CRS patients.

There is insufficient data to show that a large volume is more effective than a nasal spray.

The addition of xylitol, sodium hyaluronate, and xyloglucan to nasal saline irrigation may have a positive effect. The addition of baby shampoo, honey, or dexpanthenol as well as higher temperature and higher salt concentration do not confer additional benefit.

The steering group advises the use of nasal saline irrigation with isotonic saline or Ringer's lactate with or without the addition of xylitol, sodium hyaluronate, and/or xyloglucan and advises against the use of baby shampoo and hypertonic saline solutions due to side effects.

$\begin{array}{ll}\begin{array}{l}\text { Aspirin treatment after } \\ \text { desensitization (ATAD) with oral } \\ \text { aspirin in N-ERD }\end{array} & \begin{array}{l}\text { Oral ATAD has been shown to be significantly more effective and clinically relevant than placebo in improving QOL } \\ \text { (measured with SNOT) and total nasal symptom score in patients with N-ERD. However, the change in SNOT from treating } \\ \text { with oral ATAD compared to placebo did not reach the clinically important mean difference. ATAD reduced symptoms } \\ \text { after six months compared to placebo. However, ATAD is associated with significant adverse effects, and the risks of not } \\ \text { taking the medication strictly on a daily basis puts a burden on patient and caregiver. } \\ \text { Based on these data, the EPOS2020 steering group suggests that ATAD can be a treatment for N-ERD patients with } \\ \text { CRSwNP whenever there is confidence in the patient's compliance. }\end{array} \\ \begin{array}{ll}\text { Aspirin treatment after } \\ \text { desensitization (ATAD) with nasal } \\ \text { lysine aspirin in N-ERD }\end{array} \\ \begin{array}{ll}\text { ATAD with lysine aspirin and platelet inhibitors (like Pradugrel) have not been shown to be an effective treatment in } \\ \text { Cow salicylate diet }\end{array} \\ \text { CRSWP patients with N-ERD and are not advised. }\end{array}$

Local and systemic antifungal $\quad 1 \mathrm{a}(-) \quad$ Local and systemic antifungal treatments do not have a positive effect of QOL, symptoms and signs of disease in patients treatments with CRS. The EPOS2020 steering group advises against the use of anti-mycotics in CRS.

\begin{tabular}{|c|c|c|}
\hline Anti-lgE & $\mathrm{lb}$ & $\begin{array}{l}\text { Anti-IgE therapy has been proposed as a promising biologic therapy for CRS. Two RCTs that evaluated anti-lgE monoclonal } \\
\text { antibody did not show impact on disease specific QOL but one study did show an effect on the physical domain of SF-36 } \\
\text { and AQLQ. One study demonstrated lower symptom scores (change from baseline in anti IgE group) for nasal congestion, } \\
\text { anterior rhinorrhoea, loss of sense of smell, wheeze and dyspnoea, a significant reduction of NPS on endoscopic } \\
\text { examination, and Lund-MacKay scores on radiologic imaging. Due to the small study population in the existing studies, } \\
\text { further studies with larger population sizes are needed and are underway. The available data are insufficient to advise on } \\
\text { the use of anti-IgE in CRSwNP at this moment. }\end{array}$ \\
\hline Anti-II-5 & $\mathrm{lb}$ & $\begin{array}{l}\text { There is only one large sufficiently powered study with Mepolizumab that showed a significant reduction in patients' need } \\
\text { for surgery and an improvement in symptoms. Unlike in CRS, there is a significant experience with anti-II5 in other type } \\
2 \text { driven diseases like asthma that do show a favourable safety profile so far. The EPOS2020 steering group advises use of } \\
\text { mepolizumab in patients with CRSwNP fulfilling the criteria for treatment with monoclonal antibodies (when approved). }\end{array}$ \\
\hline
\end{tabular}

Anti IL-4/IL-13 (IL-4 receptor a) la At the moment the only anti-II-4 treatment studied in CRS is dupilumab. Dupilumab is the only monoclonal antibody that is approved for the treatment of CRSWNP so far. When evaluating all trials with dupilumab, the drug seems to induce conjunctivitis in trials in patients with atopic dermatitis but not in trials with asthma and CRSwNP. No other adverse events have been reported in the literature until now. The EPOS steering group advises to use dupilumab in patients with CRSwNP fulfilling the criteria for treatment with monoclonal antibodies.

Probiotics

$1 b(-)$

Muco-active agents
Although probiotic therapies show theoretical promise, the two studies performed so far did not show any differences compared to placebo. For this reason, the EPOS2020 steering group advises against the use of probiotics for the treatment of patients with CRS.

Data on the effect of muco-active agents in CRS are very limited. The only DBPCT evaluating the addition of S-carboxymethylcysteine to clarithromycin showed a significantly higher percentage of patients with effective response and improved characteristics of nasal discharge at 12 weeks. The EPOS2020 steering group considered the quality of the data insufficient to advise on the use of muco-active agents in the treatment of patients with CRS.

Of five RCTs evaluating herbal treatment, a large DBPCT, using tablets, showed overall no effect, although a post-hoc sensitivity analysis, showed a significant benefit in major symptom score at 12 weeks of treatment over placebo in patients with a diagnosis of CRS for $>1$ year and a baseline MSS $>9$ (out of max 15). Of the four studies evaluating different local herbal treatment, three showed a favourable effect. However, not all studies were blinded and the quality of the studies was variable.

The treatment does not show significantly more adverse events than placebo. The quality of the evidence for the local treatment is low. Based on the available data, the EPOS2020 group cannot advise on the use of herbal medicine in CRS.

Chinese medicine
There is no evidence that traditional Chinese medicine or acupuncture is more effective than placebo in the treatment of CRS. The safety of Chinese medicine is unclear because most of the papers are not (easily) accessible. Minor and serious adverse events can occur during the use of acupuncture and related modalities, contrary to the common impression that acupuncture is harmless. For this reason, the EPOS2020 steering group advises against the use of traditional Chinese medicine or acupuncture. 
Table 1.6.1. Cont.

\begin{tabular}{|c|c|c|}
\hline Therapy & $\begin{array}{l}\text { Level of } \\
\text { evidence }\end{array}$ & GRADE recommendation \\
\hline Oral verapamil & $1 b$ & $\begin{array}{l}\text { A very small pilot study showed significant improvement in QOL (SNOT-22), polyp score (VAS), and CT scan (LM-score) of } \\
\text { oral verapamil over placebo. (Potential) side effects limited the dosage. } \\
\text { The quality of the evidence for oral verapamil is very low. Based on the potential side effects the EPOS2020 steering group } \\
\text { advises against the use of oral verapamil. }\end{array}$ \\
\hline Nasal furosemide & $1 b$ & $\begin{array}{l}\text { A recent DBPCT study showed significantly reduced QOL (SNOT-22) scores and polyp score (VAS), and significantly } \\
\text { more patients with an NPS of } 0 \text { in the furosemide nasal spray treated group versus placebo. There was no indication of a } \\
\text { difference in adverse events between topical furosemide and placebo. However, the quality of the evidence is very low. } \\
\text { The EPOS2020 steering group cannot advise on the use of nasal furosemide. }\end{array}$ \\
\hline Capsaicin & $1 b$ & $\begin{array}{l}\text { Capsaicin showed a significant decrease in nasal obstruction and nasal polyp score in two small studies, however data on } \\
\text { other symptoms like rhinorrhea and smell are either non-significant or unreported. The quality of the evidence is low and } \\
\text { the EPOS steering group concludes that capsaicin may be an option in treatment of CRS in patients with CRSWNP but that } \\
\text { larger studies are needed. }\end{array}$ \\
\hline Proton-pump inhibitors & $1 \mathrm{~b}(-)$ & $\begin{array}{l}\text { Proton-pump inhibitors have been shown in one study to be not effective. Moreover, long term use of proton pump } \\
\text { inhibitors has been associated with increased risk of cardiovascular disease. The EPOS2020 steering group therefore does } \\
\text { advise against the use of proton pump inhibitors in the treatment of CRS. }\end{array}$ \\
\hline Bacterial lysate & $1 b$ & $\begin{array}{l}\text { There is one DBPCT from } 1989 \text { comparing the bacterial lysate Broncho-Vaxom to placebo in a large group of CRS patients } \\
\text { resulting in a significant decrease in purulent nasal discharge and headache over the full six month period compared to } \\
\text { placebo and reduced opacification of the sinus X-ray. Based on this limited evidence, the EPOS2020 steering group cannot } \\
\text { advise on the use of Broncho-Vaxom in the treatment of CRS. }\end{array}$ \\
\hline Phototherapy & $1 b(-)$ & $\begin{array}{l}\text { We identified two trials with opposing findings. The quality of the evidence for the use of phototherapy in patients with } \\
\text { CRS is very low. Based on the evidence, the EPOS2020 steering group cannot make a recommendation on the use of } \\
\text { phototherapy in patients with CRS. }\end{array}$ \\
\hline Filgastrim (r-met-HuG-CSF) & $1 \mathrm{~b}(-)$ & $\begin{array}{l}\text { There is one study evaluating Filgastrim compared to placebo in CRS. There was no significant difference in effect on QOL } \\
\text { between the two groups. Based on the evidence, the EPOS2020 steering group cannot make a recommendation on the } \\
\text { use of Filgastrim in patients with CRS. }\end{array}$ \\
\hline Collodial silver nasal spray & $1 \mathrm{~b}(-)$ & $\begin{array}{l}\text { One very small study did not find differences between nasal colloidal silver spray and placebo. Based on the evidence, the } \\
\text { EPOS2020 steering group cannot make a recommendation on the use of collodial silver nasal spray in patients with CRS. }\end{array}$ \\
\hline
\end{tabular}

ATAD, Aspirin treatment after desensitisation ; $\mathrm{Cl}$, confidence interval; CRS, chronic rhinosinusitis; CRSsNP, chronic rhinosinusitis without nasal polyps; CRSWNP, chronic rhinosinusitis with nasal polyps; DBPCT, double blind placebo controlled trial; LK, Lund Kennedy; MFNS, mometasone fuorate nasal spray; MSS, major symptom score; N-ERD, NSAID-exacerbated respiratory disease; NPS, nasal polyp score; QOL, quality of life; RCT, randomised controlled trial; SNOT-22, sino-nasal outcome test-22; SMD, standard mean difference.

\subsubsection{New treatment options with biologicals (monoclonal antibodies)}

The acceptance of dupilumab (anti IL-4Ra) for the treatment of CRSwNP by the US Food and Drug Administration(FDA) and European Medicines Agency (EMA) in 2019 has significantly changed the treatment options in type 2 type CRS and it is expected that other monoclonal antibodies will follow. Until 2019 monoclonal antibodies could only be prescribed in patients with concomitant (severe) asthma. Within the EUFOREA setting, the positioning of biologics in the ICP of CRS with criteria for use and stopping of biologics have been published $^{(101)}$. The EPOS2020 steering group made some modifications and tightening of these criteria. They concluded that biologicals are indicated in a patient with bilateral polyps, who had had sinus surgery or was not fit for surgery and who had three of the following characteristics: evidence of type 2 disease (tissue eosinopils $\geq 10 /$ HPF or blood eosinophils $\geq 250$ OR total lgE $\geq 100$ ), need for at least two courses of systemic corticosteroids or continuous use of systemic corticosteroids
( $\geq 2$ courses per year OR long term ( $>3$ months) low dose steroids OR contraindication to systemic steroids), significantly impaired quality of life ( SNOT-22 $\geq 40$ ), anosmic on smell test and/or a diagnosis of comorbid asthma needing regular inhaled corticosteroids (Figure 1.6.3.).

The response criteria for biologicals have been taken from the EUFOREA paper (Figure 1.6.4.), although the EPOS2020 group also discussed whether there was an indication to repeat surgery in patients on biologicals to give them a better starting point. It was decided that we had insufficient data to advise on surgery whilst on biologicals before deciding that they are not effective and that this is a research need.

\subsubsection{Conclusion}

EPOS2020 provides a full evidence based systematic review of the management of CRS that has been incorporated into an integrated care pathway (Figures 1.6.1. and 1.6.2.). A significant shift in the management of CRS has occurred since EPOS2012. The options of biologicals in the treatment of type 2 CRS will be 
a paradigm shift in the management of the disease. The exact positioning of this presently very expensive treatment needs to be determined. (Figures 1.6.3. and 1.6.4.).EPOS2020 further emphasizes the criteria for (revision) surgery in the disease.

\subsection{Paediatric chronic rhinosinusitis}

\subsubsection{Epidemiology and predisposing factors}

This section has been considerably expanded, reflecting new literature. The prevalence of CRS in paediatric patients is now estimated to be up to $4 \%{ }^{(109)}$. Both passive and active cigarette smoking are associated with chronic rhinitis and rhinosinusitis in children ${ }^{(110)}$ though a clear and definitive causal relationship between allergic rhinitis and CRS has not been established ${ }^{(111)}$. Evidence suggests that the adenoids may act as a reservoir for pathogenic bacteria, rather than a source of obstruction ${ }^{1122}$ ${ }^{113)}$ whilst the relationship between GORD and CRS in children remains controversial ${ }^{(114)}$. A large database study suggests a significant familial risk associated with paediatric $\mathrm{CRS}^{(115)}$ but studies on monozygotic twins have not shown that both siblings always develop polyps, indicating that environmental factors are as likely as genetic ones to influence the occurrence of nasal polyps.

\subsubsection{Inflammatory mechanisms}

Multiple studies suggest upregulation of different inflammatory substances important in adaptive and innate immunity as well as tissue remodelling in sinus tissues, adenoids, nasal lavage, mucus and serum in children with CRS. Although the evidence is still scarce, these studies suggest a role for inflammatory mechanisms in paediatric CRS. Although many of the markers parallel those seen in adults, the data is very heterogeneous and does not yet lend itself to endotyping. Inflammatory cytokines are present in sinus tissues of children with CRS and are more abundant when concomitant asthma is present ${ }^{(116)}$. Although more evidence is emerging to support upregulation of inflammatory markers in paranasal sinus tissues and nasal lavages of children with CRS, the data is also relatively limited and heterogeneous and again does not yet lend itself to endotyping.

\subsubsection{Management of paediatric CRS including integrated care pathway}

Medical therapy remains the mainstay of management of paediatric chronic rhinosinusitis (Table 1.7.1.). Saline nasal irrigation is recommended for the treatment of CRS in children. Addition of nasal antibiotics to saline irrigations is not recommended. There is currently no evidence to support treatment of children with CRS with either oral or intravenous antibiotics. There is also no evidence to support the utilization of prolonged macrolide therapy in children with CRS.

Intranasal steroids are recommended for use in children with CRS despite the absence of good level evidence. This is based on safety in children and favourable efficacy data in adults with CRS (see chapter 6) and children with rhinitis ${ }^{(117)}$.

There is hardly any scientific support for other ancillary therapies such as antihistamines (intranasal or oral), leukotriene modifiers, decongestants (intranasal or oral), or mucus thinners and these treatments are not recommended. Exceptions are using ancillary therapies when indicated for concomitant disease such as allergic rhinitis or GORD.

Surgical intervention is considered for patients with CRS who have failed appropriate medical therapy (and, less commonly, in complicated acute rhinosinusitis). It seems that adenoidectomy with / without antral irrigation is certainly the simplest and safest first procedure to consider in younger children with symptoms of CRS. Evidence suggests that antral irrigation should be considered in addition to an adenoidectomy in

Table 1.7.1. Evidence supporting therapy of CRS in children.

\begin{tabular}{|c|c|c|}
\hline Therapy & $\begin{array}{l}\text { Level of } \\
\text { evidence }\end{array}$ & GRADE recommendation \\
\hline Antibiotics & $1 \mathrm{~b}(-)$ & $\begin{array}{l}\text { There is no high level evidence to support the efficacy of either short or long term antibiotics for } \\
\text { CRS in children. }\end{array}$ \\
\hline Nasal corticosteroids & 5 & $\begin{array}{l}\text { There is no evidence regarding the efficacy of intranasal steroids in the treatment of CRS in } \\
\text { children. Nevertheless the EPOS steering group is supportive of their use in light of their anti- } \\
\text { inflammatory effects and excellent safety record in children. }\end{array}$ \\
\hline Systemic Steroids & $1 \mathrm{~b}(+)$ & $\begin{array}{l}\text { Adding a taper course of systemic steroids to an antibiotic (not effective on its own) is more } \\
\text { effective than placebo in the treatment of paediatric CRS. Judicious use of this regimen is } \\
\text { advised considering systemic side effects. }\end{array}$ \\
\hline Saline Irrigation & $\mathrm{lb}(+)$ & $\begin{array}{l}\text { There are a few clinical trials demonstrating the efficacy of saline irrigations in paediatric patients } \\
\text { with CRS. The EPOS steering group is supportive of the use of saline in light of the excellent } \\
\text { safety record in children. }\end{array}$ \\
\hline Adenoidectomy & 4 & $\begin{array}{l}\text { Adenoidectomy is effective in younger children with symptoms of CRS. The EPOS steering group } \\
\text { supports adenoidectomy in young children refractory to appropriate medical therapy. }\end{array}$ \\
\hline FESS & 4 & $\begin{array}{l}\text { FESS is safe and effective for the treatment of older children with CRS refractory to medical } \\
\text { therapy or previous adenoidectomy. }\end{array}$ \\
\hline
\end{tabular}

CRS, chronic rhinosinusitis; FESS, functional endoscopic sinus surgery. 
Figure 1.7.1. Integrated care pathway in paediatric CRS.

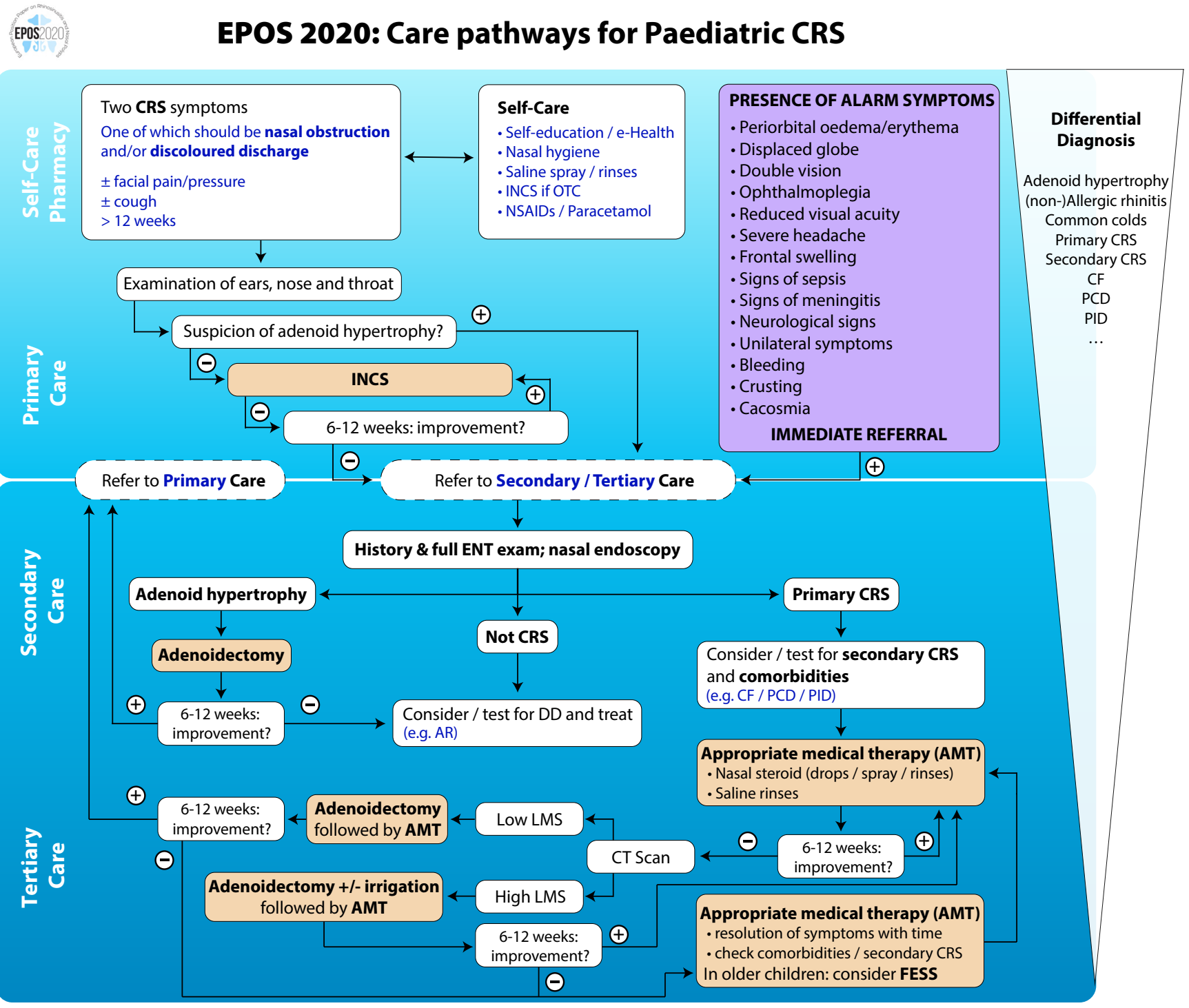

AMT, appropriate medical treatment; CF, cystic fibrosis; CRS, chronic rhinosinusitis; CT, computed tmography; DD, differential diagnosis ; INCS, intranasal corticsteroids; LMS, Lund-Mackay score; NSAIDs, non-steroidal anti-inflammatory drugs; OTC, over the counter; PCD, primary ciliary dyskinesia; PID, primary immune deficiencies.

children with asthma who have more severe disease on preoperative CT scans. FESS is a safe and possibly effective surgical modality in children with CRS and can be used as primary modality or after failure of adenoidectomy in older children. Decisions on use depends on severity of disease, age and existing co-morbidities. The rate of major complications following paediatric FESS was $0.6 \%$, and the rate of minor complications $2 \%$.

The systematic review of the literature resulted in an integrated care pathway for paediatric CRS (Figure 1.7.1.). The differential diagnosis in primary care is broad with the most essential diagnosis in young children being adenoid hypertrophy / adenoiditis. In secondary and tertiary care, the ICP also advises saline irrigation and INCS as first line treatment followed by adenoidectomy with or without sinus irrigation if insufficient.
FESS is reserved for older children who fail adenoidectomy (with sinus irrigation). CRS in children may be an indication of severe diseases such as immunodeficiencies, cystic fibrosis or primary ciliary dyskinesia. Practitioners should be aware of these and also of serious complications needing immediate referral.

\subsection{Concomitant diseases in chronic rhinosinusitis}

Chapter 8 discusses the role of concomitant diseases in CRS. The role of allergy, including central compartment atopic disease, immunodeficiencies and their role in CRS, a work-up for ENTs before referring to immunologists, lower airway disease including asthma, cystic fibrosis and PCD, fungal rhinosinusitis, vasculitis and granulomatous diseases and their role in CRS are all discussed. 
Figure 1.8.1. An overview of the interaction of fungi and the human immune response.

EPOS

Fungi and the human immune response

Allergic fungal
Fungal ball
Invasive fungal rhinosinusitis

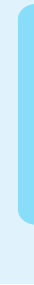

\subsubsection{Role of allergy and chronic rhinosinusitis}

It has become clear in recent years that the role of allergy in CRS depends on different phenotypes / endotypes of CRS. In some phenotypes / endotypes such as AFRS or central compartment atopic disease, allergy seems to play an important role whilst in others the prevalence does not seem to be higher than in the general population, although even in these patient groups, allergy can be an aggravating factor. Allergic rhinitis (AR) is a highly prevalent disease and there is a significant overlap in symptomatology between CRS and AR. It is not always easy to evaluate the role of sensitization to allergens in patients with CRS especially in perennial sensitisations. Optimal treatment of the allergic rhinitis seems advisable.

\subsubsection{Immunodeficiencies and their role in CRS}

Conditions that are associated with immunodeficiency are of clinical importance to rhinologists because some patients who present with CRS are predisposed to their condition by an underlying immunodeficient state. Immunodeficiency conditions may cause CRS patients to respond less favourably to standard therapies, and some patients require specific treatment for their immunodeficiencies in order for their CRS to be optimally treated.

Testing of immune function in all patients who present with CRS is almost certainly unwarranted as it is likely to produce more false positive results than true positives. However, it is recommended that recalcitrance to standard treatments (and particularly rapid recurrence of symptoms after stopping antibiotics) and association of CRS with lower respiratory tract infections (pneumonia, particularly if recurrent, or bronchiectasis) are used to identify those patients who warrant some form of immune testing.

For CRS patients suspected of having humoral immunodeficiency because of the characteristics of their presentation or their response to treatment, measurement of serum immunoglobulin levels is the key investigation. If the levels are normal, but the suspicion of humoral immunodeficiency is high, referral to a clinical immunologist is recommended.
The best approach for confirming a diagnosis of an antibodydeficiency disorder is the measurement of serum-specific antibody titres (usually lgG) in response to vaccine antigens. This approach involves immunizing a patient with protein antigens (e.g. tetanus toxoid) and polysaccharide antigens (e.g. pneumococcus) and assessing pre- and post-immunization antibody levels.

Treatment of patients with primary immune deficiency may consist of long-term antibiotics, often at half dose, pneumococcal vaccinations and immunoglobulin replacement therapy.

The prevalence of secondary immune deficiency is rising due to the increased use of immunosuppressive agents such as rituximab, corticosteroids and other drugs and otorhinolaryngologists need to directly ask about immunosuppressive agents in their history taking.

\subsubsection{Lower airway disease including asthma in relation to CRS}

Given the epidemiologic and pathophysiologic connection between CRS and lower respiratory airway disease ${ }^{(11,118)}$ the concept of global airway disease has gained more interest, leading to better diagnosis and therapeutic approaches in patients with global airway disease. Lower airway inflammation often co-exists in CRS, with up to two thirds of patients with CRS affected by comorbid asthma, COPD or bronchiectasis. Endoscopic sinus surgery in asthma has been reported to improve multiple clinical asthma parameters with improved overall asthma control, reduced frequency of asthma attacks and number of hospitalizations, as well as decreased use of oral and inhaled corticosteroids.

\subsubsection{Cystic fibrosis}

Cystic fibrosis (CF) is a life-shortening genetic condition caused by a mutation in the cystic fibrosis transmembrane conductance regulator (CFTR) gene leading to defective chloride channels, which results in secretions with more than double the viscosity of secretions of a non-CF individuals. In the Western world national screening programs on specific genetic disorders 
including CF have been implemented for newborns. Bilateral nasal polyposis in children may be a clinical indicator of CF. A major goal in the treatment of patients with $\mathrm{CF}$ is thus to prevent or delay chronic lung infections. There is a high concordance of bacteria cultured from the paranasal sinuses (based on irrigations, swabs, or mucosal biopsies) and from the lungs.

The treatment of CF is currently symptomatic whilst the treatment of the underlying genetic defect, thus curing the disease, has not yet been possible. However, new treatment options such as (the combination of) Ivacaftor, a CFTR potentiator, and Tezacaftor, a selective CFTR corrector, have shown promising results in improving rhinologic QOL in patients with CF.

Several studies have evaluated the effect of sinus surgery on pulmonary function with divergent conclusions. Sinus surgery is recommended in CF patients without chronic lung infection or with a transplanted lung in an attempt to eradicate gramnegative bacteria in the paranasal sinuses, thereby avoiding or preventing re-colonisation of the lungs. Detecting gramnegative sinus bacteria at an early stage is an important step towards eradicating the bacteria and avoiding a chronic bacterial sinus infection. The use of topical antibiotics correlates with improvement in symptom and endoscopic scoring and is safe.

\subsubsection{Primary ciliary dyskinesia}

Primary ciliary dyskinesia (PCD) is a collection of rare inherited disorders that affects motile cilia and is primarily inherited in an autosomal recessive manner. Situs inversus (i.e. Kartagener syndrome) exists in approximately half of all PCD cases. Both men and women diagnosed with PCD commonly present with fertility disorders as the reproductive process is largely dependent on ciliary function. PCD has a strong association with history of CRS, being associated with CRSwNP in 15-30\% of patients, and is commonly seen in children with CRS. PCD also predisposes to bacterial infections commonly including $\mathrm{H}$. influenza, S. pneumoniae and P. aeruginosa. In the absence of hard clinical and paraclinical criteria for diagnosing PCD, confirming the diagnosis with clinical exam alone is a challenge. An electron microscopic analysis of cilia can yield valuable information about ciliary ultrastructure and function. However, it should be noted that cilia may appear normal in patients that present with symptoms strongly suggestive of PCD due to mutations that can result in normal structure.

A number of studies have shown that exhaled nitric oxide (NO), particularly nasal NO production levels, are low in PCD patients. An nNO cut-off value of $<77 \mathrm{nl} / \mathrm{min}$ can allow detection of PCD with a sensitivity and specificity of $98 \%$ and $>99 \%$, respectively, after excluding CF and acute viral respiratory infections. Prolonged macrolide therapy has been shown to produce marked improvement in symptomatology of PCD due to the anti-inflammatory and immune-mediating properties of the antibiotic. Surgical intervention (ESS) may be required when medical therapy has failed.

\subsubsection{Fungal rhinosinusitis}

Fungi are ubiquitous in our environment and with dedicated assessments they can be found in nasal mucus from almost all healthy and diseased sinuses. However, there are several forms of sinus disease that are associated with fungi as pathogens. In these situations, rather than the fungi determining the disease process, it is usually the host immune state that determines the clinical presentation (Figure 1.8.1).

There was much prior debate regarding the role of fungi in CRSwNP. Some authors had proposed that a response to fungi might be the basis for most type 2 dominated polypoid forms of CRS. However, subsequent research has not supported this ${ }^{(119,}$ ${ }^{120)}$. Thus, this chapter will discuss these three phenotypes of 'fungal' related CRS but an intentional focus is made on AFRS as a unique phenotype, and its treatment, within the broader definition of CRS.

A fungal ball is a non-invasive collection of fungal debris. Recent studies indicate that anatomic variants are not major contributors to their formation, which in the maxillary sinus is more often related to dental interventions ${ }^{(121-123)}$. Neoosteogenesis of the maxillary sinus wall is common with fungal balls compared to normal patients and is independent of bacterial coinfection ${ }^{(124)}$. Isolated maxillary or sphenoid sinus opacification is a marker of neoplasia in $18 \%$ and malignancy in $7-10 \%$ of patients presenting with these radiologic findings so clinicians should be wary of conservative management and have a low threshold for early surgical intervention ${ }^{(125)}$. Little has changed in the management of fungal balls since 2012 which remains surgical, consisting of removal via an adequate antrostomy. However, persistent dysfunction of the sinus cavity with mucostasis was reported to be as high as $18 \%$ and, therefore, some authors have proposed a medial maxillectomy for some maxillary cases ${ }^{(126)}$. Invasive fungal rhinosinusitis (IFRS) is almost always associated with immunocompromise, of which diabetes (50\%) and haematologic malignancy (40\%) account for $90 \%$ of the immunosuppression reported ${ }^{(127)}$. IFRS is defined as any state in which fungal hyphae can be seen 'within' the mucosal tissue, demonstrating classic angio-invasion or other infiltrative patterns ${ }^{(128)}$ which result in thrombosis, tissue infarction and necrosis. Although originally several forms of invasive disease were described: granulomatous, chronic and fulminant, they all potentially represent an immunocompromised host reaction to the fungus ${ }^{(129)}$. The most common causative pathogens remain the Zygomycetes (Rhizopus, Mucor, Rhizomucor) and the Aspergillus species. Unilateral disease on radiology is typical ${ }^{(130,131)}$ but loss of contrast enhancement on MRI is more sensitive (86\%) than CT $(69 \%)$ in detecting invasive fungal disease ${ }^{(132)}$. Serum analysis via PCR (serum or whole blood) and/or galactomannan for invasive aspergillosis can be useful ${ }^{(133)}$.

There are three principles for treatment:

1. Systemic antifungals therapy should be started;

2. Patients should undergo, at least, endoscopic surgical debridement of necrotic sinonasal tissue, which may need to be repeated; 
3. The patient's immune suppression should be reduced when feasible.

Allergic fungal rhinosinusitis (AFRS) is a subset of polypoid chronic rhinosinusitis that is characterized by the presence of eosinophilic mucin with non-invasive fungal hyphae within the sinuses and a type I hypersensitivity to fungi. The EPOS2020 steering group discussed whether the term 'eosinophilic fungal rhinosinusitis' would be a better umbrella term but it was agreed that 'allergic fungal rhinosinusitis' should be retained as the principle term due to common usage, recognising that not all cases have evidence of an allergic reaction to fungi. AFRS accounts for about $5-10 \%$ of CRS cases $^{(134)}$.

Ideally all five of the major criteria in the original Bent-Kuhn diagnostic criteria should be met to make the diagnosis as three of the five are common in most cases of CRSWNP. These major criteria consist of the following ${ }^{(135)}$ :

1. Nasal polyposis;

2. Fungi on staining;

3. Eosinophilic mucin without fungal invasion into sinus tissue;

4. Type I hypersensitivity to fungi and;

5. Characteristic radiological findings with soft tissue differential densities on CT scanning and unilaterality or anatomically discrete sinus involvement.

The minor criteria include bone erosion, Charcot Leyden Crystals, unilateral disease, peripheral eosinophilia, positive fungal culture and the absence of immunodeficiency or diabetes ${ }^{(136)}$. CT shows densely packed hyperdensities in the sinuses with expansion and erosion of the bony walls whereas on MRI signal voids occur on both T1 and T2 sequences ${ }^{(137)}$. The mainstay of treatment remains surgery as medical treatment alone is usually ineffective. However, oral steroids both pre- and postoperatively are of benefit ${ }^{(138)}$. Nebulised topical corticosteroids reduce recurrence ${ }^{(139)}$ and allergen immunotherapy was also helpful in atopic individuals but studies are retrospective and underpowered. There is some evidence that oral antifungals may reduce recurrence but do not improve symptoms.

Fungal rhinosinusitis remains an important phenotype of CRS in its invasive and non-invasive forms. Clinicians should have a low threshold for seeking its diagnosis, especially in the presence of the immunocompromised. The mainstay of treatment remains surgical though may be combined with medical therapies in invasive and allergic forms. See Figure 1.6.2. which includes an integrated care pathway for AFRS although the steering group realized that diagnosis in primary and secondary care can be difficult.

\subsubsection{Vasculitis}

ANCA-associated vasculitis includes GPA, EGPA and microscopic polyangiitis (MPA) and frequently affect the upper respiratory tract and specifically the sinonasal region where they may be mistaken for more common forms of chronic rhinosinusitis.
Classically GPA affects the nose, lungs and kidneys but can present in any system and limited forms of the disease are recognised. Two thirds of patients initially present with an ENT-related symptom, of which the majority are rhinological. During the course of the disease, the majority of GPA patients experience nasal symptoms with patients experiencing crusting (75\%), discharge (70\%), nasal stuffiness (65\%), bleeding (59\%), reduced sense of smell $(52 \%)$ and facial pain $(33 \%)^{(140,141)}$. ANCA tests have become the mainstay of diagnosis in vasculitis. A positive c-ANCA test and proteinase-3 (PR3) will confirm the clinical diagnosis of GPA in up to $95 \%$ of patients with active systemic disease. An ANCA test should be considered in any patient with suspicious clinical manifestations, in particular nasal crusting and bleeding, especially if they feel disproportionally unwell(142).

Cocaine abuse in the form of nasal 'snorting' can resemble the sinonasal symptoms of GPA and can give c-ANCA and PR-3 positivity, making differentiation between the conditions difficult ${ }^{(143)}$. Without treatment the mean survival of systemic GPA is five months. Modern immunosuppressive treatment following a strategy of combined remission, induction and maintenance has markedly improved this to a mean survival of 21.7 years from diagnosis assisted by higher awareness and earlier diagnosis. Nasal irrigation, topical intranasal corticosteroid sprays or creams e.g. triamcinolone and/or a nasal lubricant such as $25 \%$ glucose and glycerine drops, honey ointment or an aqueous gel are usually recommended together with regular debridement of the crusts. The possible aetiological role of Staphylococcus aureus has led to the use of long-term oral co-trimoxazole (trimethoprim-sulfamethoxazole) and topical anti-staphylococcal creams in the nose. Reconstructive surgery has a very limited role and is associated with poor outcomes, increased scarring and adhesions so should be a last resort. Eosinophilic granulomatosis with polyangiitis (EGPA) (previously Churg Strauss Syndrome) is a rare form of vasculitis characterised by adult onset asthma, severe rhinitis, nasal polyps and other systemic manifestations as a result of widespread eosinophilic granulomatous infiltration of tissues ${ }^{(144)}$. EGPA should be considered in any patient with severe nasal polyposis who is not responding to conventional therapy. Active EGPA is characterised by marked peripheral eosinophilia (usually $>1500$ cells/ul or $>10 \%$ ) and ANCA-positivity is found in a proportion of the patients. In most patients, disease control is achieved with immunosuppressant therapy, usually oral prednisolone +/cytotoxic drugs such as pulsed cyclophosphamide, azathioprine, mycophenolate mofetil and methotrexate dependent on the severity of the disease at presentation. Sarcoidosis is a chronic multi-system inflammatory disease of unknown aetiology characterised by non-caseating granuloma. There is no definitive test for sarcoidosis other than a positive biopsy. Blood tests may include raised serum and urinary calcium levels, raised alkaline phosphatase and raised serum angiotensinconverting enzyme (SACE) but none are diagnostic (sensitivity $60 \%$; specificity $70 \%$ ). Systemic steroids remain the mainstay of treatment in sarcoidosis, though hydroxychloroquine, steroid- 
sparing cytotoxic agents such as methotrexate and TNF-alpha antagonists such as infliximab are being used.

\subsection{Patient participation, prediction, precision medicine and implementation}

\subsubsection{Patient participation in CRS}

Patient participation in rhinosinusitis can relate on an individual basis to participation of the patient in the design and/or discussion of the treatment plan, or to participation in the follow-up after medical or surgical treatment ${ }^{(145)}$. There is limited research on the impact of patient participation on outcomes of treatment in CRS.

Patient involvement, moreover, is recognized as a key component of clinical practice guideline development with important implications for guideline implementability ${ }^{(146)}$. Aspects of patient participation are covered for the first time in EPOS2020 because patient involvement is essential in the development of their future care. Patients were actively involved in the development of EPOS2020. Recent mobile health initiatives to educate patients on CRS, on correct medication use and treatment options have been implemented in certain areas in Europe with success ${ }^{(147)}$. Whilst they allow a more proactive follow-up of patients with remote monitoring of symptoms by physicians ${ }^{(147,148)}$ the impact of e-health on CRS outcomes still needs to be defined and proven.

For individual patients, shared decision-making is one of the four cardinal principles of Precision Medicine ${ }^{(149)}$. In order to improve compliance, it is important to explain the aim of ongoing usage or any maintenance treatments to both control symptoms and reduce need for recurrent interventions. Information on the safety of treatment and instructions for use must be provided in all necessary languages. While physicians are likely to understand the chronic nature of sinus disease in many patients and the need for ongoing treatment, it is essential to share this with the patient from the outset. The aim of treatment is to achieve adequate control of symptoms with as little need for intervention as possible; for many this will involve ongoing usage of intranasal treatments and in some, repeated need for systemic treatments or surgical interventions. Some patients will remain inadequately controlled despite receiving optimum current evidence-based care. Cure, with an absence of symptoms in the setting of no ongoing medication usage, is unusual in CRS with the exception of localized sinus disease where there has been a curable cause, such as an odontogenic source.

\subsubsection{Primary, secondary and tertiary disease prevention in CRS}

Prevention may be considered as primary, secondary and tertiary ${ }^{(150)}$. Primary prevention aims to reduce incidence of disease by reducing exposure to risk factors or triggers. CRS is a heterogeneous disease, where inflammation, mucociliary dysfunction and changes in the microbial community interact with differing influences to cause disease; the aetiology is likely multifactorial, and opportunities to prevent targeting specific causes will likely vary between subgroups. Occupational and environmental factors, especially exposure to tobacco smoke, are of increasing importance in primary prevention and the effects of global warming should be carefully monitored. Co-morbidities such as allergy, asthma and GORD should be considered. Genetic and microbiological factors will likely become of greater importance. Early diagnosis and selection of the optimal treatment is central to secondary prevention. Optimising medical treatment and consideration of the timing and extent of surgery can improve outcomes. In tertiary prevention, a careful review of ongoing treatment, technique and compliance with medication should be undertaken. Growth in digital healthcare and patient apps may encourage self-management and increase compliance. There are a small number of studies using big data sets that suggest that endoscopic sinus surgery for CRS reduces the yearly incidence of new asthma diagnoses. Those patients who have later surgery may develop higher rates of asthma than those who undergo surgery at an earlier timepoint. Finally, the prevention of recurrent disease is important. Continued use of intranasal corticosteroids after surgery has been shown to improve postoperative endoscopic scores in all CRS patients and, in those with CRSwNP, reduce risk of recurrence. Adherence with prescribed postoperative medications dropped to only $42 \%$ at 12 months after surgery in one study, despite regular telephone contact; strategies to improve this such as utilizing digital technology will likely be important in future. One can also imagine that other forms of ensuring the application of postoperative medication, e.g. by drug eluting stents, may solve the problem of compliance. A small number of studies have found that ongoing occupational exposure to irritants may increase risk of recurrence. Any factors thought to be involved in the underlying aetiology of CRS in each individual patient should be addressed where possible to reduce risk of recurrence. In contrast to the large number of studies evaluating changes in HRQOL after treatment, few studies have evaluated patient satisfaction with outcomes of treatment, and only following surgical interventions. Although data is limited, it appears that pre-treatment counselling to ensure that a patient has realistic expectations of treatment outcomes is important to avoid a dissatisfied patient. This is in respect to improvement overall and in those symptoms deemed to be most important to the patient, as well as optimizing outcomes with respect to their nasal symptoms.

\subsubsection{Prediction}

There are no studies evaluating the natural history of untreated CRS although there is some evidence for the adverse effects of delayed surgical treatment ${ }^{(151)}$. Notwithstanding ethical considerations, there is clearly an urgent need for more research in this area. Similarly, there are very few studies predicting outcomes of medical treatment. When predicting outcomes following sinus surgery, a number of studies have shown that the preoperative symptom score such as SNOT-22 is the best predictor of outcome ${ }^{(152,153)}$. Primary surgery has better outcomes than revision. When loss of smell is a major symptom, response in olfactory function to oral corticosteroids (OCS) predicts the outcome of surgery. Prediction of recurrent 
disease involves many factors including age, gender, ethnicity, co-morbidities, and duration of disease. Both blood and tissue eosinophil levels can be measured with little additional expense and may be used to help predict risk of recurrence and need for targeted postoperative care.

\subsubsection{Precision medicine}

In 2015 President Obama launched the precision medicine initiative: "delivering the right treatment at the right time, every time, to the right person". The principles of precision medicine can be implemented within existing adult treatment algorithms for $\mathrm{CRS}^{(149)}$. At the time of diagnosis, prediction of success of the initiated treatment as well as patient participation in decisions regarding the treatment plan can be undertaken. Precision medicine allows real-time clinical decision support at the point of care with implementation of harmonized care based on quality criteria and allows patients to be treated and monitored more precisely and effectively to better meet their individual needs. It brings together clinicians from many inter-related specialities, scientists and above all patients in a collaborative effort to provide the most efficient and effective management.

\subsubsection{Implementation}

The implementation of high-quality guidelines and position papers is essential to improve clinical practice and public health. We tried to make EPOS2020 implementable by writing a clear and concise executive summary with extensive chapters with all the evidence behind it. We hope that the executive summary will be translated in all necessary languages. Furthermore, we reached out to many key opinion leaders all over the world to review and comment on the document and included their suggestions in the final text. We do realize that not all advice in EPOS2020 can be followed in all health care systems and social circumstances. A full implementation plan will be written separately to the EPOS2020 document in the near future.

\subsection{Pharmacist perspective on rhinosinusitis}

Chapter 10 gives the pharmacist's perspective on rhinosinusitis and offers specific advice to pharmacists on how to differentiate and treat the various forms of ARS (common cold, post-viral rhinosinusitis and acute bacterial rhinosinusitis) and CRS in contradistinction to allergic rhinitis. Special emphasis has been placed on the avoidance of antibiotics in the treatment of rhinosinusitis and the role that the pharmacist can have in advising patients on the correct use of nasal sprays.

\subsection{Research priorities in rhinosinusitis}

Chapter 11 gives an overview of research priorities. In many areas of rhinosinusitis, evidence is still of low quality and most subchapters in EPOS2020 originally ended with:'more research is needed to provide high quality evidence.' We decided, therefore, to remove the majority of these exortations and to collate the most urgent questions in this final chapter.

\subsection{Methods used in EPOS2020}

In chapter 12 the methods used in EPOS2020 are discussed. We describe the development strategy used in EPOS2020 has been published before we started the work ${ }^{(155)}$. We did a full systematic review of the literature and used GRADE methodology for recommendations. On a large number of practical clinical questions with no or or very low level of evidence we conducted a Delphi exercise.

\section{References}

1. Fokkens W, Lund V, Mullol J. European position paper on rhinosinusitis and nasal polyps 2007. Rhinol Suppl. 2007:1-136.

2. Fokkens W, Lund V, Bachert C, et al. European position paper on rhinosinusitis and nasal polyps. Rhinol Suppl. 2005:1-87

3. Fokkens WJ, Lund VJ, Mullol J, et al. European Position Paper on Rhinosinusitis and Nasal Polyps 2012. Rhinol Suppl. 2012;23:3 p preceding table of contents, 1-298.

4. Hastan D, Fokkens WJ, Bachert C, et al. Chronic rhinosinusitis in Europe--an underestimated disease. A GA(2)LEN study. Allergy 2011;66:1216-23.

5. Hirsch AG, Stewart WF, Sundaresan AS, et al. Nasal and sinus symptoms and chronic rhinosinusitis in a population-based sample. Allergy 2017;72:274-81.

6. Obaseki D, Potts J, Joos G, et al. The relation of airway obstruction to asthma, chronic rhinosinusitis and age: results from a population survey of adults. Allergy 2014;69:1205-14.

7. Sundaresan AS, Hirsch AG, Storm M, et al. Occupational and environmental risk factors for chronic rhinosinusitis: a systematic review. Int Forum Allergy Rhinol. 2015;5:996-1003.

8. Piatt Jr. JH. Intracranial suppuration compli- cating sinusitis among children: an epidemiological and clinical study. J Neurosurg Pediatr 2011;7:567-74.

9. Jaume F, Quintó L, Alobid I, Mullol J. Overuse of diagnostic tools and medications in acute rhinosinusitis in Spain: a population-based study (the PROSINUS study). BMJ open 2018;8:e018788.

10. Wei B, Liu F, Zhang J, et al. Multivariate analysis of inflammatory endotypes in recurrent nasal polyposis in a Chinese population. Rhinology 2018;56:216-26.

11. Tomassen P, Vandeplas G, Van Zele T, et al. Inflammatory endotypes of chronic rhinosinusitis based on cluster analysis of biomarkers. J Allergy Clin Immunol. 2016;137:1449-56. e4.

12. Jarvis D, Newson R, Lotvall J, et al. Asthma in adults and its association with chronic rhinosinusitis: the GA2LEN survey in Europe. Allergy. 2012:67:91-8

13. Hakansson K, Thomsen SF, Konge $L$, Mortensen J, Backer V, von Buchwald C. A comparative and descriptive study of asthma in chronic rhinosinusitis with nasal polyps. Am J Rhinol Allergy. 2014;28:383-7.

14. Samitas $\mathrm{K}$, Carter A, Kariyawasam HH, Xanthou G. Upper and lower airway remod- elling mechanisms in asthma, allergic rhinitis and chronic rhinosinusitis: The one airway concept revisited. Allergy 2018;73:993-1002.

15. van der Veen J, Seys SF, Timmermans M, et al. Real-life study showing uncontrolled rhinosinusitis after sinus surgery in a tertiary referral centre. Allergy 2017;72:282-90.

16. Snidvongs K, Heller GZ, Sacks R, Harvey RJ. Validity of European position paper on rhinosinusitis disease control assessment and modifications in chronic rhinosinusitis. Otolaryngol Head Neck Surg. 2014;150:47986.

17. Calus L, Van Bruaene N, Bosteels C, et al. Twelve-year follow-up study after endoscopic sinus surgery in patients with chronic rhinosinusitis with nasal polyposis. Clin Transl Allergy. 2019;9:30.

18. Stjärne P, Odebäck P, Ställberg B, Lundberg J, Olsson P. High costs and burden of illness in acute rhinosinusitis: real-life treatment patterns and outcomes in Swedish primary care. Primary Care Resp J. 2012;21:174-9.

19. Remenschneider AK, Scangas G, Meier JC, et al. EQ-5D-derived health utility values in patients undergoing surgery for chronic rhinosinusitis. Laryngoscope 2015;125:1056-61.

20. Gliklich RE, Metson R. The health impact of 
chronic sinusitis in patients seeking otolaryngologic care. Otolaryngol Head Neck Surg. 1995;113:104-9.

21. Teul I, Zbislawski W, Baran S, Czerwinski F, Lorkowski J. Quality of life of patients with diseases of sinuses. J Physiol Pharmacol. 2007;58 Suppl 5:691-7.

22. Garbutt J, Spitznagel E, Piccirillo J. Use of the modified SNOT-16 in primary care patients with clinically diagnosed acute rhinosinusitis. Arch Otolaryng Head Neck Surg 2011;137:792-7.

23. Hopkins C, Browne JP, Slack R, et al. The national comparative audit of surgery for nasal polyposis and chronic rhinosinusitis. Clinical Otolaryngol. 2006;31:390-8.

24. Teul I, Baran S, Zbislawski W. Upper respiratory tract diseases in self-evaluation of health status of Polish students based on the SF-36 questionnaire. J Physiol Pharmacol. 2008;59 Suppl 6:697-707.

25. Dietz de Loos DA, Hopkins C, Fokkens WJ. Symptoms in chronic rhinosinusitis with and without nasal polyps. Laryngoscope 2013;123:57-63.

26. Abdalla S, Alreefy H, Hopkins C. Prevalence of sinonasal outcome test (SNOT-22) symptoms in patients undergoing surgery for chronic rhinosinusitis in the England and Wales National prospective audit. Clin Otolaryngol 2012;37:276-82.

27. Bhattacharyya N, Lee LN. Evaluating the diagnosis of chronic rhinosinusitis based on clinical guidelines and endoscopy. Otolaryngol Head Neck Surg. 2010;143:147-51.

28. Bhattacharyya N. Contemporary assessment of the disease burden of sinusitisThe economic burden and symptom manifestations of chronic rhinosinusitis. Am J Rhinol Allergy. 2009;23:392-5.

29. Wahid NW, Smith R, Clark A, Salam M, Philpott C. The Socioeconomic Cost of Chronic Rhinosinusitis Study. Rhinology 2020;in press.

30. Lourijsen ES, Fokkens WJ, Reitsma S. Direct and indirect costs of Dutch adult patients with Chronic Rhinosinusitis with nasal polyps. Rhinology 2020;in press.

31. Bhattacharyya N. Assessing the additional disease burden of polyps in chronic rhinosinusitis. Annals Otol, Rhinol Laryngol 2009:118:185-9.

32. Gross Mark;Burgess LP, Rick; Sheridan. Endoscopic Sinus Surgery Complications in Residency. Laryngoscope 1997;107:1080-5.

33. Gliklich RE, Metson R. Economic implications of chronic sinusitis. Otolaryngol Head Neck Surg. 1998;118:344-9.

34. Bhattacharyya N, Orlandi RR, Grebner J, Martinson M. Cost burden of chronic rhinosinusitis: a claims-based study. Otolaryngol Head Neck Surg. 2011;144:440-5.

35. Blackwell DL, Collins JG, Coles R. Summary health statistics for U.S. adults: National Health Interview Survey, 1997. Vital Health Stat. 10 2002:1-109.

36. Goetzel RZ, Hawkins K, Ozminkowski RJ, Wang S. The health and productivity cost burden of the "top 10" physical and mental health conditions affecting six large U.S. employers in 1999. J Occup Environ Med. 2003:45:5-14.

37. Rudmik L. Economics of Chronic Rhinosinusitis. Curr Allergy Asthma Reports 2017;17:20.

38. Hoffmans R, Wagemakers A, van Drunen C, Hellings $P$, Fokkens W. Acute and chronic rhinosinusitis and allergic rhinitis in relation to comorbidity, ethnicity and environment. PloS one 2018;13:e0192330.

39. Revai K, Dobbs LA, Nair S, Patel JA, Grady JJ, Chonmaitree T. Incidence of acute otitis media and sinusitis complicating upper respiratory tract infection: the effect of age. Pediatrics 2007;119:e1408-12.

40. Benninger MS, Ferguson BJ, Hadley JA, et al. Adult chronic rhinosinusitis: Definitions, diagnosis, epidemiology, and pathophysiology. Otolaryngol Head Neck Surg. 2003;129:S1-32.

41. Shapiro DJ, Gonzales R, Cabana MD, Hersh AL. National trends in visit rates and antibiotic prescribing for children with acute sinusitis. Pediatrics. 2011;127:28-34.

42. Rosenfeld RM, Piccirillo JF, Chandrasekhar SS, et al. Clinical practice guideline (update): Adult sinusitis. Otolaryngol Head Neck Surg. 2015;152:S1-S39.

43. Leung R, Almassian S, Kern R, Conley D, Tan B, Chandra R. Patient level decision making in recurrent acute rhinosinusitis: a cost-benefit threshold for surgery. Laryngoscope. 2013;123:11-6.

44. Alkire BC, Bhattacharyya N. An assessment of sinonasal anatomic variants potentially associated with recurrent acute rhinosinusitis. Laryngoscope. 2010;120:631-4.

45. Jain R, Stow N, Douglas R. Comparison of anatomical abnormalities in patients with limited and diffuse chronic rhinosinusitis. Int Forum Allergy Rhinol. 2013;3:493-6.

46. Loftus PA, Lin J, Tabaee A. Anatomic variants of the paranasal sinuses in patients with recurrent acute rhinosinusitis. Int Forum Allergy Rhinol. 2016;6:328-33.

47. Costa ML, Psaltis AJ, Nayak JV, Hwang PH. Medical therapy vs surgery for recurrent acute rhinosinusitis. Int Forum Allergy Rhinol. 2015;5:667-73

48. De Sario M, Katsouyanni K, Michelozzi P. Climate change, extreme weather events, air pollution and respiratory health in Europe. Eur Resp Journal. 2013, 826-43.

49. Kuiper JR, Hirsch AG, Bandeen-Roche K, et al. Prevalence, severity, and risk factors for acute exacerbations of nasal and sinus symptoms by chronic rhinosinusitis status. Allergy. 2018;73:1244-53.

50. Eyigor $\mathrm{H}$, Basak S. [Evaluation of predisposing factors and bacteriologic agents in pediatric rhinosinusitis]. Kulak Burun Bogaz Ihtis Derg 2005;15:49-55.

51. Pant H, Ferguson BJ, Macardle PJ. The role of allergy in rhinosinusitis. Curr Opin Otolaryngol Head Neck Surg. 2009;17:232-8.

52. Flook EP, Kumar BN. Is there evidence to link acid reflux with chronic sinusitis or any nasal symptoms? A review of the evidence. Rhinology. 2011;49:11-6.

53. Seresirikachorn K, Snidvongs K, Chitsuthipakorn W, et al. EPOS2012 has better specificity com-pared to IDSA2012 for diagnosing acute bacterial rhinosinusitis. Rhinology. 2018;56:241-4.

54. Pilan RR, Pinna FR, Bezerra TF, et al. Prevalence of chronic rhinosinusitis in Sao Paulo. Rhinology. 2012;50:129-38.

55. Shi JB, Fu QL, Zhang H, et al. Epidemiology of chronic rhinosinusitis: results from a cross-sectional survey in seven Chinese cities. Allergy. 2015;70:533-9.

56. Dietz de Loos D, Lourijsen ES, Wildeman MAM, et al. Prevalence of chronic rhinosinusitis in the general population based on sinus radiology and symptomatology. J Allergy Clin Immunol. 2019;143:1207-14.

57. Tomassen P, Newson RB, Hoffmans R, et al. Reliability of EP3OS symptom criteria and nasal endoscopy in the assessment of chronic rhinosinusitis--a GA(2) LEN study. Allergy. 2011;66:556-61.

58. Hirsch AG, Nordberg C, Bandeen-Roche K, et al. Radiologic sinus inflammation and symptoms of chronic rhinosinusitis in a population-based sample. Allergy. 2019, 10.1111/ all.14106.

59. Hamizan AW, Loftus PA, Alvarado R, et al. Allergic phenotype of chronic rhinosinusitis based on radiologic pattern of disease. Laryngoscope. 2018;128:2015-21.

60. Philpott CM, Erskine S, Hopkins C, et al. Prevalence of asthma, aspirin sensitivity and allergy in chronic rhinosinusitis: data from the UK National Chronic Rhinosinusitis Epidemiology Study. Respiratory research 2018;19:129.

61. De Schryver E, Derycke L, Campo P, et al. Alcohol hyper-responsiveness in chronic rhinosinusitis with nasal polyps. Clin Exp Allergy 2016, 10.1111/cea.12836.

62. Endam LM, Filali-Mouhim A, Boisvert P, Boulet $L-P$, Bosse $Y$, Desrosiers M. Genetic variations in taste receptors are associated with chronic rhinosinusitis: a replication study. Int forum All Rhinol. 2014;4:200-6.

63. Purnell PR, Addicks BL, Zalzal HG, et al. Single Nucleotide Polymorphisms in Chemosensory Pathway Genes GNB3, TAS2R19, and TAS2R38 Are Associated with Chronic Rhinosinusitis. Int Arch Allergy Immunol. 2019;180:72-8.

64. Stevens WW, Peters AT, Tan BK, et al. Associations Between Inflammatory Endotypes and Clinical Presentations in Chronic Rhinosinusitis. J Allergy Clin Immunol In Practice. 2019, 10.1016/j. jaip.2019.05.009.

65. Brook CD, Kuperstock JE, Rubin SJ, Ryan MW, Platt MP. The association of allergic sensitization with radiographic sinus opacification. Am J Rhinol Allergy 2017;31:12-5.

66. Hummel T, Whitcroft KL, Andrews P, et al. Position paper on olfactory dysfunction. Rhinology Supplement 2017;54:1-30.

67. Stogbauer J, Wirkner K, Engel C, et al. Prevalence and risk factors of smell dysfunc- 
tion - a comparison between five German population-based studies. Rhinology. 2019, 10.4193/Rhin19.181.

68. Landis BN, Hummel T. New evidence for high occurrence of olfactory dysfunctions within the population. Am J Med. 2006;119:91-2.

69. Lane AP, Turner J, May L, Reed R. A genetic model of chronic rhinosinusitis-associated olfactory inflammation reveals reversible functional impairment and dramatic neuroepithelial reorganization. J Neurosci. 2010;30:2324-9.

70. Pfaar O, Landis BN, Frasnelli J, Huttenbrink KB, Hummel T. Mechanical obstruction of the olfactory cleft reveals differences between orthonasal and retronasal olfactory functions. Chem Senses. 2006;31:27-31.

71. Jones NS. Sinogenic facial pain: Diagnosis and management. Otolaryngol Clin North Am. 2005;38:1311-25.

72. Kirsch CFE, Bykowski J, Aulino JM, et al. ACR Appropriateness Criteria((R)) Sinonasal Disease. J Am Coll Radiol 2017;14:S550-9.

73. Younis RT, Anand VK, Davidson B. The role of computed tomography and magnetic resonance imaging in patients with sinusitis with complications. Laryngoscope 2002;112:224-9.

74. Bhattacharyya N. A comparison of symptom scores and radiographic staging systems in chronic rhinosinusitis. American J of Rhinology 2005;19:175-9.

75. Lund VJ, Kennedy DW. Staging for rhinosinusitis. Otolaryng - Head \& Neck Surg. 1997;117:S35-40.

76. Bayonne E, Kania R, Tran P, Huy B, Herman P. Intracranial complications of rhinosinusitis. A review, typical imaging data and algorithm of management*. Rhinology. 2009;47:59-65.

77. Lund VJ, Mackay IS. Staging in rhinosinusitus. Rhinology. 1993;31:183-4.

78. Orlandi RR, Kingdom TT, Hwang PH, et al. International Consensus Statement on Allergy and Rhinology: Rhinosinusitis. Int Forum Allergy Rhinol. 2016;6 Suppl 1:S22S209.

79. Amine $M$, Lininger $L$, Fargo KN, Welch KC. Outcomes of endoscopy and computed tomography in patients with chronic rhinosinusitis. Int Forum Allergy Rhinol. 2013;3:73-9.

80. Daramola OO, Lidder AK, Ramli R, et al. Patient knowledge and perception of computed tomography scan in the management of chronic rhinosinusitis symptoms. Laryngoscope. 2015;125:791-5.

81. Leung RM, Chandra RK, Kern RC, Conley DB, Tan BK. Primary care and upfront computed tomography scanning in the diagnosis of chronic rhinosinusitis: A cost-based decision analysis. Laryngoscope. 2014;124:12-8.

82. Bulla S, Blanke P, Hassepass F, et al. Reducing the radiation dose for low-dose CT of the paranasal sinuses using iterative reconstruction: Feasibility and image quality. Eur J of Radiology. 2012;81:2246-50.

83. Sodickson A. CT radiation risks coming into clearer focus. BMJ. 2013;346:f3102-f.

84. Fraczek M, Guzinski M, Morawska-Kochman $M$, Krecicki T. Investigation of sinonasal anat- omy via low-dose multidetector CT examination in chronic rhinosinusitis patients with higher risk for perioperative complications. Eur Arch of Oto-Rhino-Laryngol. 2017;274:787-93.

85. Gevaert P, Van Bruaene N, Cattaert T, et al. Mepolizumab, a humanized anti-IL-5 mAb, as a treatment option for severe nasal polyposis. J of Allergy and Clin. Imm. 2011;128:988-9.

86. Pinto JM, Mehta N, DiTineo M, Wang J, Baroody FM, Naclerio RM. A randomized, double-blind, placebo-controlled trial of anti-lgE for chronic rhinosinusitis. Rhinology. 2010;48:318-24.

87. Hopkins C, Hettige R, Soni-Jaiswal A, et al. CHronic Rhinosinusitis Outcome MEasures (CHROME), developing a core outcome set for trials of interventions in chronic rhinosinusitis. Rhinology. 2018;56:22-32.

88. 88. Kim DH, Seo Y, Kim KM, Lee S, Hwang SH. Usefulness of Nasal Endoscopy for Diagnosing Patients With Chronic Rhinosinusitis: A Meta-Analysis. Am J Rhinol Allergy. 2019, 10.1177/1945892419892157:19 45892419892157.

89. Flores Kim J, McCleary N, Nwaru Bl, Stoddart A, Sheikh A. Diagnostic accuracy, risk assessment, and cost-effectiveness of component-resolved diagnostics for food allergy: A systematic review. Allergy. 2018;73:1609-21.

90. Eiringhaus K, Renz H, Matricardi P, Skevaki C. Component-Resolved Diagnosis in Allergic Rhinitis and Asthma. J Appl Lab Med 2019;3:883-98

91. Lotsch J, Hummel T. A data science-based analysis of seasonal patterns in outpatient presentations due to olfactory dysfunction. Rhinology. 2019, 10.4193/Rhin19.099.

92. Rimmer J, Hellings P, Lund VJ, et al. European position paper on diagnostic tools in rhinology. Rhinology. 2019;57:1-41.

93. Kern RC. Chronic sinusitis and anosmia: pathologic changes in the olfactory mucosa. Laryngoscope. 2000;110:1071-7.

94. Apter AJ, Gent JF, Frank ME. Fluctuating olfactory sensitivity and distorted odor perception in allergic rhinitis. Arch Otolaryngol Head Neck Surg. 1999;125:1005-10.

95. Hsieh JW, Keller A, Wong M, Jiang R-S, Vosshall LB. SMELL-S and SMELL-R: Olfactory tests not influenced by odor-specific insensitivity or prior olfactory experience. Proc of the Nat Academy of Sciences. 2017:114:11275-84.

96. Leite SHP, Jain R, Douglas RG. The clinical implications of computerised fluid dynamic modelling in rhinology. Rhinology. 2019;57:29.

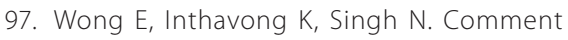
on the European position paper on diagnostic tools in rhinology - computational fluid dynamics. Rhinology. 2019, 10.4193/ Rhin 19.269.

98. Rimmer J. Reply to the letter by Wong et al. Rhinology. 2019, 10.4193/Rhin19.296.1.

99. Snidvongs K, Lam M, Sacks R, et al. Structured histopathology profiling of chronic rhinosinusitis in routine practice. Int Forum All
Rhinology. 2012;2:376-85.

100.Jiang N, Kern RC, Altman KW Histopathological evaluation of chronic rhinosinusitis: a critical review. Am J Rhinol Allergy. 2013;27:396-402.

101. Fokkens WJ, Lund V, Bachert C, et al. EUFOREA consensus on biologics for CRSwNP with or without asthma. Allergy. 2019;74:2312-9.

102. Hoggard M, Zoing M, Biswas K, Taylor MW, Douglas RG. The sinonasal mycobiota in chronic rhinosinusitis and control patients. Rhinology. 2019;57:190-9.

103. Bachert C, Han JK, Desrosiers M, et al. Efficacy and safety of dupilumab in patients with severe chronic rhinosinusitis with nasal polyps (LIBERTY NP SINUS-24 and LIBERTY NP SINUS-52): results from two multicentre, randomised, double-blind, placebo-controlled, parallel-group phase 3 trials. Lancet. 2019;394:1638-50.

104. Bachert C, Sousa AR, Lund VJ, et al. Reduced need for surgery in severe nasal polyposis with mepolizumab: Randomized trial. J Allergy Clin Immunol. 2017;140:1024-31.e14.

105. Rudmik L, Soler ZM, Hopkins C, et al. Defining appropriateness criteria for endoscopic sinus surgery during management of uncomplicated adult chronic rhinosinusitis: a RAND/UCLA appropriateness study. Int Forum Allergy Rhinol. 2016;6:557-67.

106. Beswick DM, Mace JC, Rudmik L, Soler ZM, DeConde AS, Smith TL. Productivity changes following medical and surgical treatment of chronic rhinosinusitis by symptom domain. Int Forum Allergy Rhinol. 2018;8:1395-405.

107. Kowalski ML, Agache I, Bavbek S, et al. Diagnosis and management of NSAIDExacerbated Respiratory Disease (N-ERD)-a EAACI position paper. Allergy. 2018, 10.1111/ all.13599.

108. Oakley GM, Christensen JM, Sacks R, Earls $P$, Harvey RJ. Characteristics of macrolide responders in persistent post-surgical rhinosinusitis. Rhinology. 2018;56:111-7.

109. Sidell D, Shapiro NL, Bhattacharyya N. Obesity and the risk of chronic rhinosinusitis, allergic rhinitis, and acute otitis media in school-age children. Laryngoscope. 2013;123:2360-3.

110. Reh DD, Higgins TS, Smith TL. Impact of tobacco smoke on chronic rhinosinusitis: A review of the literature. Int. Forum of Allergy and Rhinol. 2012, 362-9.

111. Georgalas C, Vlastos I, Picavet V, van Drunen C, Garas G, Prokopakis E. Is chronic rhinosinusitis related to allergic rhinitis in adults and children? Applying epidemiological guidelines for causation. Allergy. 2014;69:828-33.

112. Neff $L$, Adil EA. What is the role of the adenoid in pediatric chronic rhinosinusitis? Laryngoscope. 2015;125:1282-3.

113. Belcher R, Virgin F. The Role of the Adenoids in Pediatric Chronic Rhinosinusitis. Med Sci. (Basel) 2019:7.

114. Brietzke SE, Shin JJ, Choi S, et al. Clinical consensus statement: pediatric chronic rhinosinusitis. Otolaryngol - Head Neck Surg. 2014;151:542-53. 
115. Orb Q, Curtin K, Oakley GM, et al. Familial risk of pediatric chronic rhinosinusitis. Laryngoscope. 2016;126:739-45.

116. Skoner DP, Anfuso A, Ramadan H, et al. Sinus and adenoid inflammation in children with chronic rhinosinusitis and asthma. Am J of Resp and Crit Care Med. 2015;191.

117. Schenkel EJ, Skoner DP, Bronsky EA, et al. Absence of growth retardation in children with perennial allergic rhinitis after one year of treatment with mometasone furoate aqueous nasal spray. Pediatrics. 2000;105:E22.

118. Yoo KH, Ahn HR, Park JK, et al. Burden of Respiratory Disease in Korea: An Observational Study on Allergic Rhinitis, Asthma, COPD, and Rhinosinusitis. Allergy Asthma Immunol Res. 2016;8:527-34.

119. Orlandi RR, Marple BF. The role of fungus in chronic rhinosinusitis. Otolaryngol Clin North Am 2010;43:531-7, viii.

120. Fokkens WJ, Lund VJ, Mullol J, et al. EPOS 2012: European position paper on rhinosinusitis and nasal polyps 2012. A summary for otorhinolaryngologists. Rhinology. 2012;50:112.

121. Oshima H, Nomura K, Sugawara M, Arakawa K, Oshima T, Katori Y. Septal deviation is associated with maxillary sinus fungus ball in male patients. Tohoku J Exp Med. 2014;232:201-6.

122. Yoon YH, Xu J, Park SK, Heo JH, Kim YM, Rha KS. A retrospective analysis of 538 sinonasal fungus ball cases treated at a single tertiary medical center in Korea (1996-2015). Int Forum Allergy Rhinol. 2017;7:1070-5.

123. Park GY, Kim HY, Min JY, Dhong HJ, Chung SK. Endodontic treatment: a significant risk factor for the development of maxillary fungal ball. Clin Exp Otorhinolaryngol. 2010;3:136-40.

124. Jun YJ, Shin JM, Lee JY, Baek BJ. Bony Changes in a Unilateral Maxillary Sinus Fungal Ball. J Craniofac Surg. 2018;29:e44-e7.

125. Knisely A, Holmes T, Barham H, Sacks R, Harvey R. Isolated sphenoid sinus opacification: A systematic review. Am J Otolaryngol. 2017:38:237-43.

126. Nomura K, Asaka D, Nakayama T, et al. Sinus fungus ball in the Japanese population: clinical and imaging characteristics of 104 cases. Int J Otolaryngol. 2013;2013:731640.

127. Turner JH, Soudry E, Nayak JV, Hwang PH. Survival outcomes in acute invasive fungal sinusitis: a systematic review and quantitative synthesis of published evidence. Laryngoscope. 2013;123:1112-8.

128. Trief D, Gray ST, Jakobiec FA, et al. Invasive fungal disease of the sinus and orbit: a comparison between mucormycosis and Aspergillus. Br J Ophthalmol 2016;100:184-8.

129. deShazo RD, O'Brien M, Chapin K, SotoAguilar M, Gardner L, Swain R. A new classification and diagnostic criteria for invasive fungal sinusitis. Arch. of Otolaryngol. -- Head Neck Surg. 1997:123:1181-8.

130. Wandell GM, Miller C, Rathor A, et al. A mul- ti-institutional review of outcomes in biopsy-proven acute invasive fungal sinusitis. Int Forum Allergy Rhinol. 2018;8:1459-68.

131. Payne SJ, Mitzner R, Kunchala S, Roland L, McGinn JD. Acute Invasive Fungal Rhinosinusitis: A 15-Year Experience with 41 Patients. Otolaryngol Head Neck Surg. 2016;154:759-64.

132.Groppo ER, El-Sayed IH, Aiken AH, Glastonbury CM. Computed tomography and magnetic resonance imaging characteristics of acute invasive fungal sinusitis. Arch Otolaryngol Head Neck Surg. 2011;137:100510.

133. Arvanitis M, Anagnostou T, Mylonakis E. Galactomannan and Polymerase Chain Reaction-Based Screening for Invasive Aspergillosis Among High-Risk Hematology Patients: A Diagnostic Meta-analysis. Clin Infect Dis. 2015;61:1263-72.

134. Bakhshaee M, Fereidouni M, Nourollahian M, Movahed R. The presence of fungal-specific $\mathrm{IgE}$ in serum and sinonasal tissue among patients with sinonasal polyposis. Eur. Arch Oto-rhino-laryng. 2014;271:2871-5.

135. Bent 3rd JP, Kuhn FA, Bent JP, Kuhn FA. Diagnosis of allergic fungal sinusitis. Otolaryngol Head Neck Surg. 1994;111:580-8.

136. deShazo RD, Swain RE. Diagnostic criteria for allergic fungal sinusitis. J Allergy Clin. Immunol. 1995;96:24-35.

137. Aribandi M, McCoy VA, Bazan C, 3rd. Imaging features of invasive and noninvasive fungal sinusitis: a review. Radiographics. 2007;27:1283-96.

138. Landsberg R, Segev Y, DeRowe A, Landau T, Khafif A, Fliss DM. Systemic corticosteroids for allergic fungal rhinosinusitis and chronic rhinosinusitis with nasal polyposis: a comparative study. Otolaryngol Head Neck Surg. 2007;136:252-7.

139. Dai Q, Duan C, Liu Q, Yu H. Effect of nebulized budesonide on decreasing the recurrence of allergic fungal rhinosinusitis. Am J Otolaryngol. 2017;38:321-4.

140. Gottschlich S, Ambrosch P, Kramkowski $D$, et al. Head and neck manifestations of Wegener's granulomatosis. Rhinology. 2006:44:227-33.

141. Srouji IA, Andrews P, Edwards C, Lund VJ. Patterns of presentation and diagnosis of patients with Wegener's granulomatosis: ENT aspects. J Laryngol Otol. 2007;121:653-8.

142. Bossuyt X, Cohen Tervaert J-W, Arimura Y, et al. Position paper: Revised 2017 international consensus on testing of ANCAs in granulomatosis with polyangiitis and microscopic polyangiitis. Nature Rev Rheumatol. 2017;13:683-92.

143. Trimarchi M, Bertazzoni G, Bussi M. Cocaine induced midline destructive lesions. Rhinology. 2014;52:104-11.

144. Greco A, Marinelli C, Fusconi M, et al. Clinic manifestations in granulomatosis with polyangiitis. Int. J of immunopath pharmacol.
2016:29:151-9

145. Hellings PW. Joint action with European CRSWNP Patients for better outcomes. Rhinology. 2019;57:321.

146. Pugin B, Deneyer L, Bachert C, et al. Patient Advisory Board for Chronic Rhinosinusitis - A EUFOREA initiative. Rhinology. 2019;57:331-5.

147. Seys SF, Bousquet J, Bachert C, et al. mySinusitisCoach: patient empowerment in chronic rhinosinusitis using mobile technology. Rhinology. 2018;56:209-15.

148. Khanwalkar AR, Shen J, Kern RC, et al. Utilization of a novel interactive mobile health platform to evaluate functional outcomes and pain following septoplasty and functional endoscopic sinus surgery. Int Forum Allergy Rhinol. 2019;9:345-51.

149. Hellings PW, Fokkens WJ, Bachert C, et al. Positioning the principles of precision medicine in care pathways for allergic rhinitis and chronic rhinosinusitis - A EUFOREAARIA-EPOS-AIRWAYS ICP statement. Allergy. 2017;72:1297-305.

150. Hopkins C, Surda P, Bast F, Hettige R, Walker A, Hellings PW. Prevention of chronic rhinosinusitis. Rhinology. 2018:56:307-15.

151. Hopkins C, Rimmer J, Lund VJ. Does time to endoscopic sinus surgery impact outcomes in Chronic Rhinosinusitis? Prospective findings from the National Comparative Audit of Surgery for Nasal Polyposis and Chronic Rhinosinusitis. Rhinology. 2015;53:10-7.

152. Hopkins C, Rudmik L, Lund VJ. The predictive value of the preoperative Sinonasal Outcome Test-22 score in patients undergoing endoscopic sinus surgery for chronic rhinosinusitis. Laryngoscope. 2015;125:1779-84.

153. Rudmik L, Soler ZM, Hopkins C. Using postoperative SNOT-22 to help predict the probability of revision sinus surgery. Rhinology. 2016;54:111-6.

154.Grayson JW, Hopkins C, Mori ES, B. Contemporary Classification of Chronic Rhinosinusitis: Moving beyond CRSWNP and CRSsNP. JAMA Otolaryngol Head Neck Surg. 2020; in press.

155. Fokkens W, Desrosiers M, Harvey R, et al. EPOS2020: development strategy and goals for the latest European Position Paper on Rhinosinusitis. Rhinology 2019;57:162-8.

\section{Wytske J. Fokkens}

Department of Otorhinolaryngology

Amsterdam Medical Center

Amsterdam

the Netherlands

E-mail:

w.j.fokkens@amsterdamumc.nl 